\title{
Proper Orthogonal Decomposition for Reduced-Order Thermal Solution in Hypersonic Aerothermoelastic Simulations
}

\author{
Nathan J. Falkiewicz* and Carlos E. S. Cesnik ${ }^{\dagger}$ \\ Department of Aerospace Engineering, University of Michigan, Ann Arbor, MI 48109, USA
}

The ability to perform full-order aerothermoelastic simulations of hypersonic vehicles is hindered by the strong coupling exhibited between the aerodynamics, heat transfer, and structural dynamic response in the hypersonic flight regime. As a result of these interactions, alternative techniques are necessary to obtain computationally tractable systems of governing equations and their solutions. This work addresses the use of proper orthogonal decomposition for reduced-order solution of the heat transfer problem within a hypersonic modeling framework. The specific challenge of handling time-dependent boundary conditions due to transient aerodynamic heating is discussed. An overview of the proper orthogonal decomposition is given and two methods for solution of the reduced system of ordinary differential equations are outlined. The methodology is applied to a representative hypersonic vehicle control surface model for two cases in which the time-history of the thermal load vector is known a priori: one in which the boundary conditions are time-independent and another in which they are time-varying. Results demonstrate the ability of the reduced-order solution to approximate the full-order solution with reasonable accuracy. Finally, a time-marching hypersonic aerothermoelastic framework is described in which proper orthogonal decomposition is used for the transient thermal solution.

\section{Nomenclature}

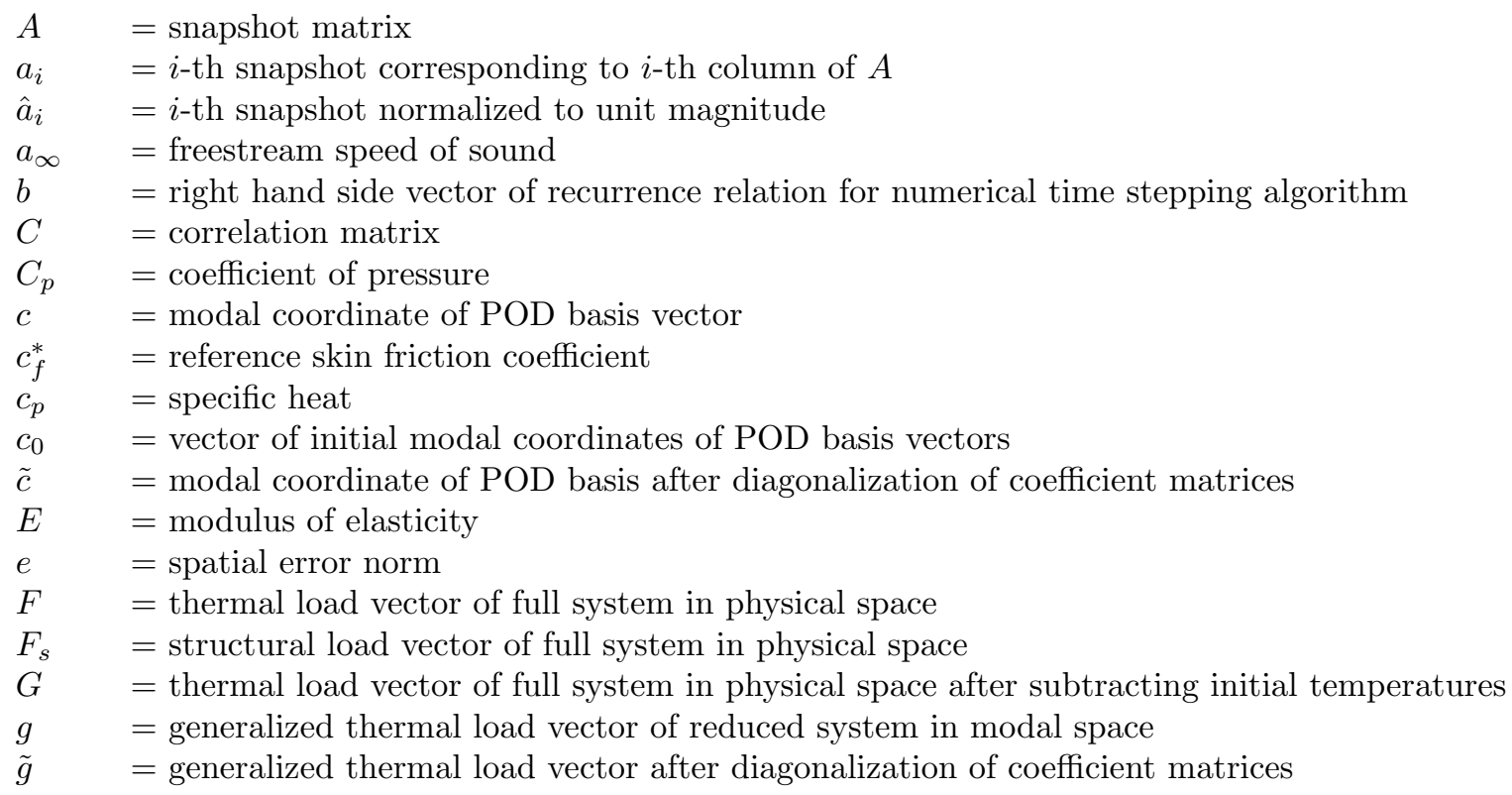

*Ph.D. Candidate, Dept. of Aerospace Engineering, 1320 Beal Ave, Ann Arbor, MI 48109, Student Member AIAA.

${ }^{\dagger}$ Professor of Aerospace Engineering, Dept. of Aerospace Engineering, University of Michigan, 1320 Beal Ave, Ann Arbor, MI 48109, Associate Fellow AIAA. 


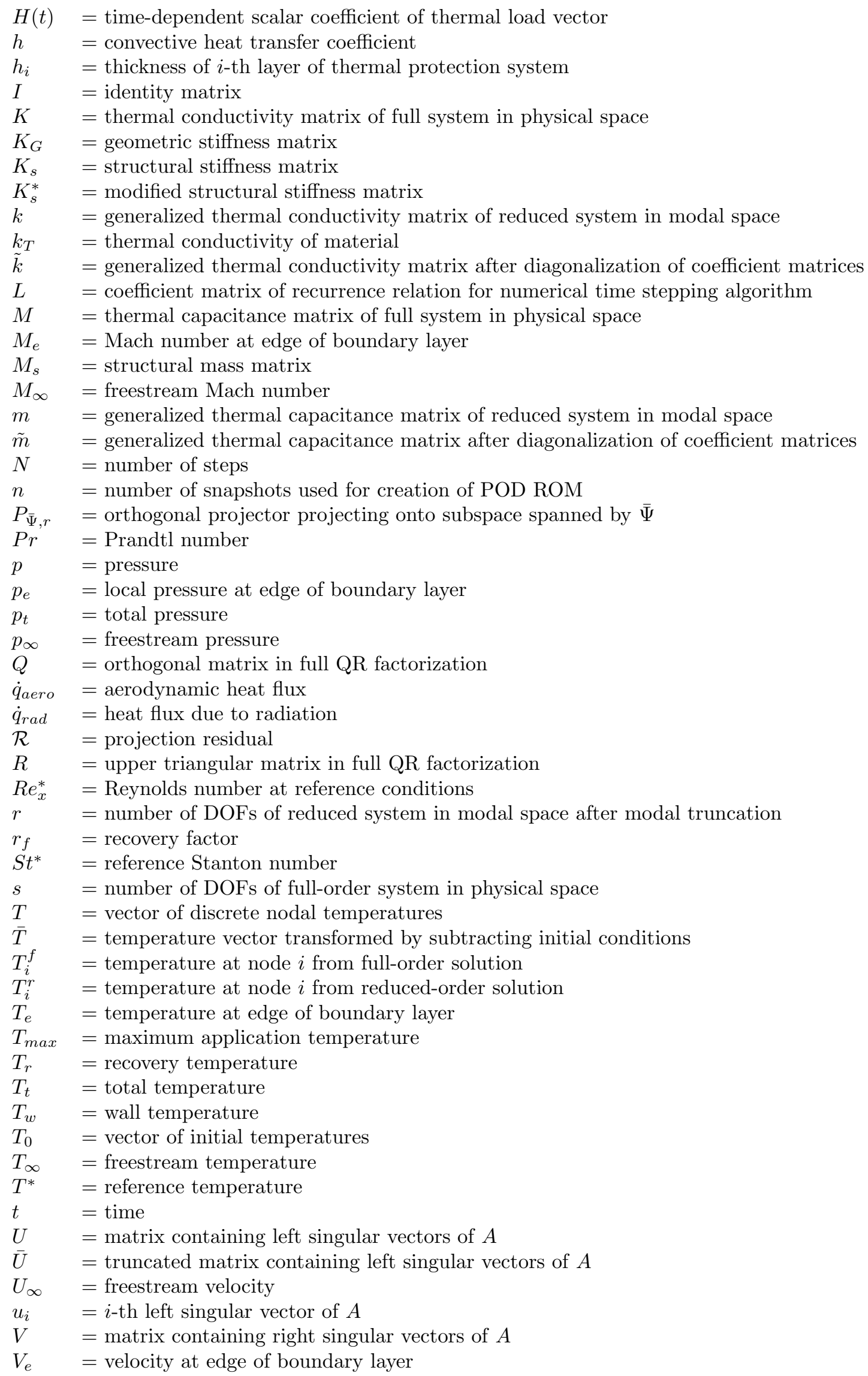




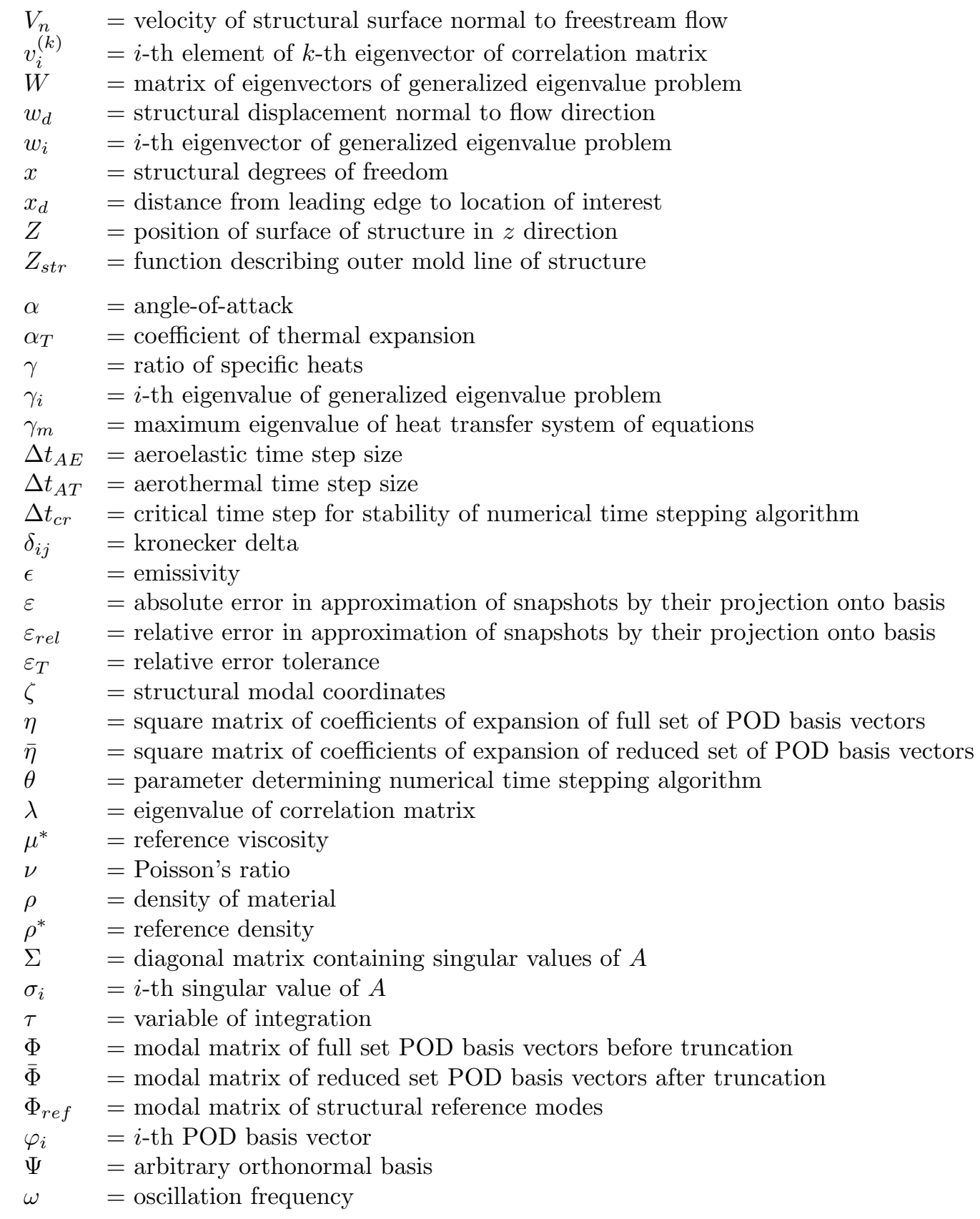

\section{Introduction}

\section{I.A. Overview of the Aerothermoelastic Problem}

Hypersonic vehicle (HSV) design and simulation require an interdisciplinary approach due to complex physics and the coupling of a variety of disciplines. The highly integrated nature of hypersonic flight stems from various factors specific to these types of vehicles. Airbreathing hypersonic vehicles typically consist of a tightly integrated airframe along with a scramjet propulsion system. The forward fuselage of the vehicle represents the compression ramp which produces the necessary flow conditions for the inlet of the propulsion system. This results in a pressure distribution which causes a nose-up pitching moment. The aft section of the vehicle consists of an external exhaust nozzle shaped to allow for expansion of the flow exiting the engine. Additionally, the location of the engine below the vehicle center of gravity results a nose-up pitching moment due to thrust which must be balanced. ${ }^{1}$ Further complicating the coupling between the propulsion system and the airframe are the elastic deformations of the forebody and vehicle pitch response which affect 
the inlet conditions to the engine. ${ }^{2}$ Thus to assess the overall vehicle performance, the effect of flexibility must be considered.

A significant aspect in the design and simulation of airbreathing hypersonic vehicles (HSVs) involves the aerodynamic heating that develops at the surface of the vehicle due to stagnation of the flow and friction within the boundary layer. ${ }^{3,4}$ Hypersonic vehicles with airbreathing propulsion systems must fly at relatively low altitudes to maintain the dynamic pressure required for optimal engine performance. ${ }^{4}$ One consequence of this requirement is that the high dynamic pressure and high Reynolds number lead to surface heating becoming a major design driver. The surface heating in turn leads to heat being conducted through the internal vehicle structure. The spatial variation of temperature throughout the structure leads to a change in stiffness distribution through two effects: degradation of material properties due to temperature-dependence and geometric stiffening effects due to internal thermal stresses. Thus, to accurately capture the structural dynamic response of the vehicle, the transient temperature distribution must be known so that the stiffness distribution can be determined.

This work will focus on the solution of the transient heat transfer problem within the context of fully coupled aerothermoelastic vehicle simulations. While specific attention will be given to the thermal aspect of the problem in this work, it cannot be considered in isolation due to the complex interactions among disciplines as described above. A schematic of the aerothermoelastic coupling mechanisms exhibited in hypersonic flight is given in Fig. 1.

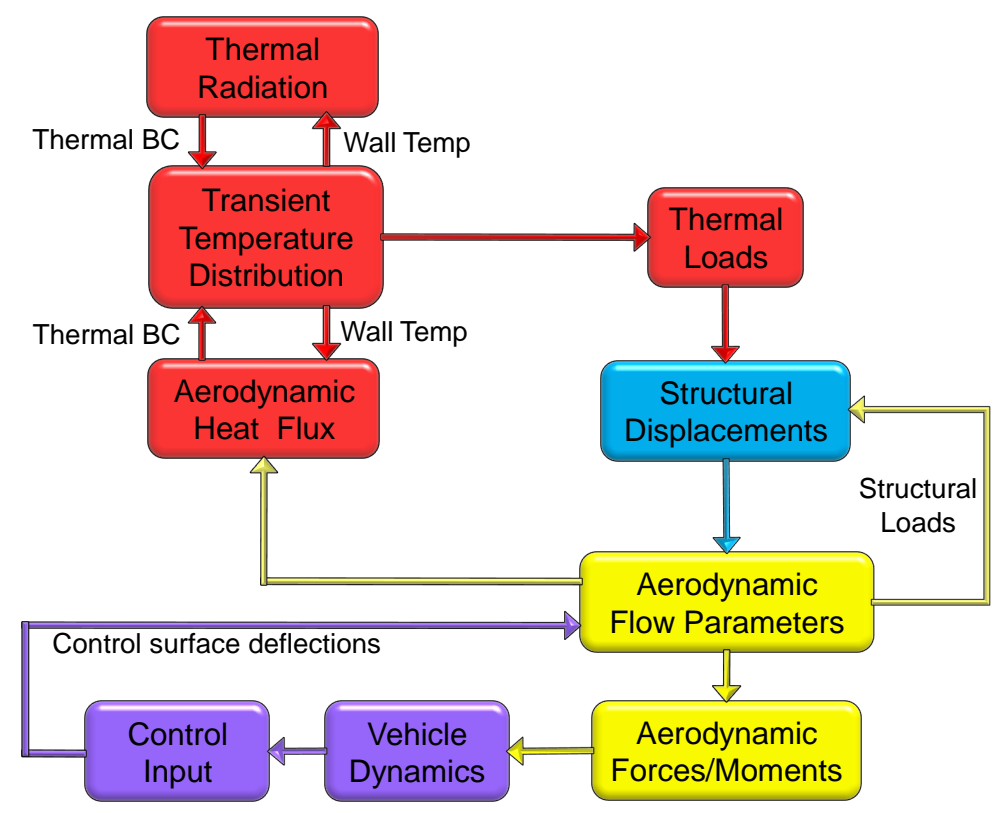

Figure 1. Coupling between aerodynamic heating, heat transfer, elastic airframe, and unsteady aerodynamics.

Stagnation effects and boundary layer friction lead to the existence of an aerodynamic heat flux at the surface of the vehicle. Note the two-way coupling between the aerodynamic heat flux and the transient temperature distribution. The upward pointing arrow illustrates the fact that the aerodynamic heat flux represents the thermal boundary condition which drives heat through the structure. The downward pointing arrow indicates the fact that the heat flux is dependent on the wall temperature of the structure. A similar two-way coupling exists between the thermal radiation boundary condition and the wall temperature. Further complicating the problem is the fact that the aerodynamic flow properties must be known in order to calculate the aerodynamic heat flux. Once the aerodynamic problem is solved and the heat flux is known, the boundary conditions for the heat transfer problem are generated. The existence of the heat flux will lead to heat being conducted through the internal structure, thus producing a transient temperature distribution throughout the vehicle structure.

The loads on the structure will have two components: thermal loads resulting from differential thermal expansion of the structure and unsteady aerodynamic pressure loads. As a result of these loads, the structure will displace relative to its undeformed configuration. As the structure deforms and the aerodynamic profile of the vehicle is modified, the aerodynamic flow properties over the vehicle change. The aerodynamic 
flow properties must be updated as the structure deforms since they will affect the loads on the structure, the aerodynamic heat flux, and the aerodynamic forces and moments on the vehicle. Once the updated aerodynamic flow parameters are known, the pressures can be integrated over the vehicle to calculate the resultant forces and moments on the vehicle. Based on the forces and moments, the vehicle equations of motion are propagated and necessary control inputs (i.e., control surface deflections) are determined based on the commanded trajectory. The control surface deflections in turn result in a change in the aerodynamic flow parameters and the process is then repeated at the next time step. Because the control surfaces are expected to provide a significant contribution to the aerodynamic lift, drag, and moments acting on the vehicle, thorough analysis of the couplings involved with such a structure is required in order to accurately predict vehicle performance and controllability. While the main thrust of this paper involves developing solution methods for the transient temperature distribution of a hypersonic vehicle, the unsteady aerodynamics, aerodynamic heating, and structural dynamics of the vehicle will also be modeled to accurately capture the boundary conditions to the thermal problem.

\section{I.B. Aerothermoelastic Modeling Framework}

As the geometry of the hypersonic vehicle structure is expected to be complex, a numerical approach to the thermal problem is taken in this work as opposed to seeking closed-form, analytical solutions. While there exist many well-established numerical techniques for solving thermal problems ${ }^{5}$ such as finite volume, spectral element, boundary element, finite element, and finite difference methods, using these methods by themselves would require solutions of large systems of equations for the large-scale structures of interest. The corresponding computational time associated with such a solution is not feasible for design and simulation within the hypersonic aerothermoelastic framework proposed here. Furthermore, the number of states that would be involved in such a solution would be impractical for use in control system design and evaluation models. As such, this work will investigate the use of a reduced-order modeling technique known as the proper orthogonal decomposition (POD) for solution of the transient thermal problem. Though full-order finite element solutions of the problem will be used to derive the reduced-order model (ROM), these solutions will be computed off-line, prior to the actual simulation. Thus once the thermal ROM is created, there is no need to return to the full-order model for high-fidelity solutions. Another advantage of this approach is the ability to tailor the level of fidelity of the ROM to attain the appropriate balance between computational complexity and accuracy. Due to the comprehensive nature of the problem, reduced-order models or firstprinciples models will also be used for the structural dynamic, aerothermal, and unsteady aerodynamic components. However these models are used solely for computational tractability and in-depth analysis of model reduction techniques is limited to that employed for the thermal aspect of the problem. An overview of the time-marching aerothermoelastic framework in which the reduced-order thermal solution will be employed is given in Fig. 2.

The process begins with the calculation of the heat flux on the outer surface of the structure at initial time. With the boundary conditions and initial conditions of the thermal problem known, the transient temperature distribution is marched forward in time. Solution of the heat transfer problem is carried out in modal space using modes from POD (described in a later section) to avoid the computational cost of running full-order finite element analysis. The bypassing of the full-order thermal solution via the reduced-order solution is indicated by the gray blocks in Fig. 2. This work will consider three coupling mechanisms between the thermal and structural solutions. The first involves the thermal stresses that will occur in the structure due to differential thermal expansion resulting from the spatially varying temperature distribution. The second is due to the temperature-dependence of the Young's modulus resulting from the high temperatures experienced in hypersonic flight. The third involves the thermal loads that are generated on the structure due to thermal expansion. With the stiffness and structural loads known, the structural dynamics system of equations in physical space is transformed to a suitable reduced modal basis to be described in a subsequent section. The reduced modal system is then solved for the modal coordinates to obtain the structural response. The structural deformations will couple with the aerothermal problem due to the effect on aerodynamic flow properties, which will change the heat flux. The deformations also result in a change in aerodynamic pressures which modify the structural loads. With the deformed configuration known at the current time step, the aerodynamic flow parameters and heat flux are recalculated and the procedure is repeated at the next time instant. 


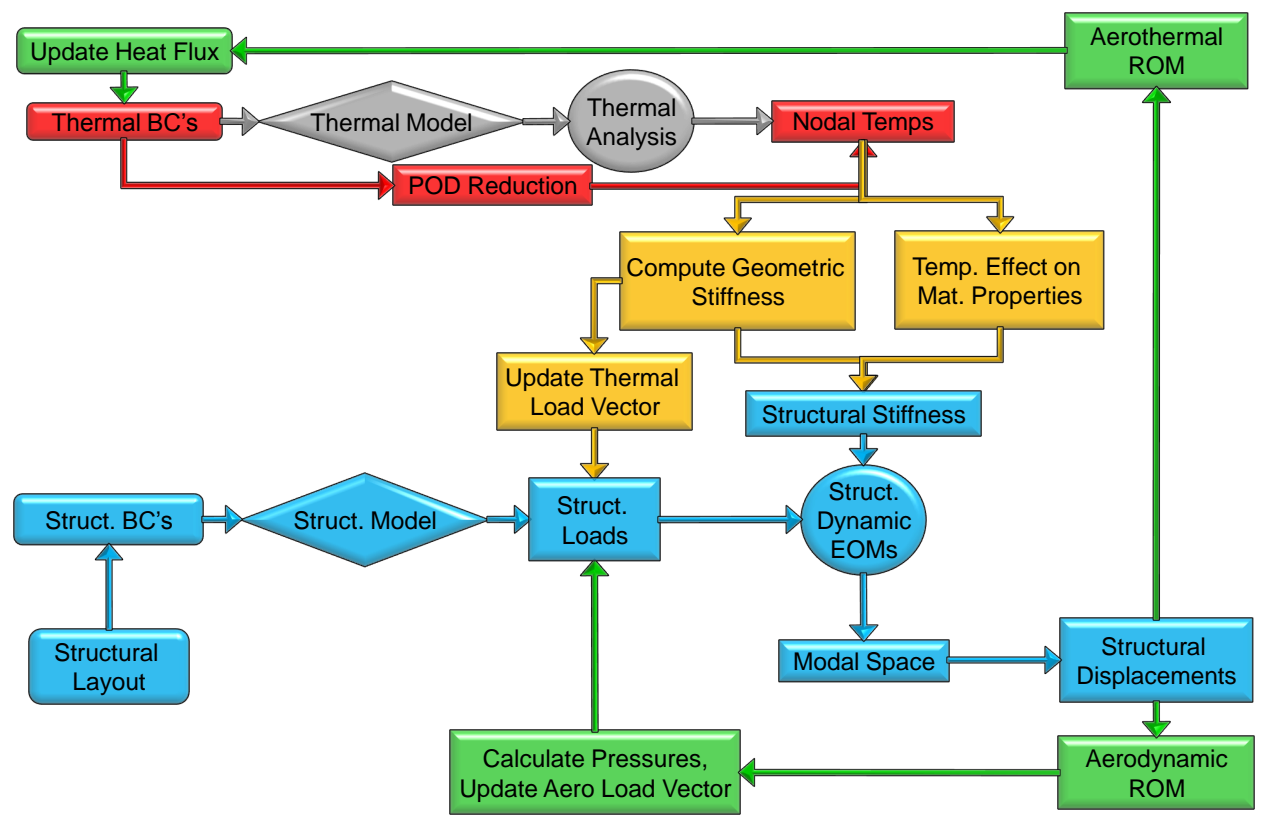

Figure 2. Reduced-order aerothermoelastic modeling framework.

\section{I.C. Prior Work on Reduced-Order Thermal Modeling}

There have been a variety of methods employed in the literature to reduce the order of thermal problems. The well known structural dynamics reduction methods of Guyan Reduction ${ }^{6}$ and component modes synthesis ${ }^{7}$ have been extended for use in both linear and nonlinear transient thermal problems. ${ }^{8-10}$ One paper ${ }^{11}$ utilized $^{-1}$ Guyan Reduction and component modes synthesis for reduction of the thermal problem in conjunction with nonlinear identification techniques for solution of the structural and fluid problems for coupled solid/fluid analysis of a turbine disk model. Results of this study showed that both Guyan reduction and component modes synthesis provided sufficient reduction of the problem for efficient computation of temperature histories at selected locations of the model. However, a disadvantage to both of these methods is that tuning of the reduced-order model was necessary to obtain the desired accuracy as the error of a particular reduced-order model cannot be determined a priori. In particular, Guyan reduction requires selection of the set of active and omitted degrees of freedom while the component modes synthesis methodology used in these works requires the selection of a subset of eigenmodes related to the set of omitted temperatures.

Another technique that has been used for reduction of both linear and nonlinear transient thermal problems is the modal identification method. ${ }^{12-15}$ The use of this technique is largely motivated by inverse heat transfer problems in which one desires to determine boundary conditions to a thermal problem based on measured temperature evolutions at selected locations. Given a state-space representation of the fullorder system, this technique seeks another state-space representation of the dynamics which is of much lower order than the original system and gives a good approximation to the output of the original system. As the reduced-order state-space representation is written in modal space, the identification of a subset of eigenmodes of the system is required. The primary advantage of the modal identification method is that no knowledge of the thermal capacitance and thermal conductivity matrices is required for calculation of the eigenmodes of the system. Rather than solving an eigenvalue problem of the full system, the eigenmodes are identified through the minimization of a quadratic criterion related to the difference between the output vector of the full-order model and that of the reduced-order model. Therefore, this method is useful for situations in which the computational cost of a large-scale eigenvalue problem cannot be afforded and for problems in which the thermal matrices of a system are unknown.

The modal identification method was developed and implemented for linear multivariable systems with multiple inputs and outputs and was shown to reduce a model of order 1643 to one of order 26 while still maintaining sufficient accuracy. ${ }^{12}$ Due to the ill-posedness of the inverse problem, computational difficulties in its solution can arise due to the ill-conditioning involved matrices. As such, a regularization procedure 
was implemented in another work ${ }^{13}$ for the reduced-order solution of a linear heat conduction system that made use of modal identification. Application of the methodology to a three-dimensional example problem showed good agreement between the full-order and reduced models for reduction from order 1331 to order 9. A subsequent two-part work extended the modal identification method for use in nonlinear systems ${ }^{14,15}$ for single-input/multi-output systems. The methodology employed in this work was similar to the one employed for linear systems in that it identifies a state-space representation of reduced order to approximate the high-fidelity system. However, for the nonlinear case additional manipulation was needed to separate the linear and nonlinear terms in the equation. This work introduced nonlinearity through a linear variation of the thermal conductivity with temperature. The methodology as applied to a three-dimensional cube demonstrated that the reduced-order model was able to obtain solutions for the inverse problem whereas the detailed model was not for the same time integration scheme.

While the modal identification method provides a significant computational advantage for certain problems such as inverse heat transfer problems, its applicability within the aerothermoelastic framework of this work is questionable. Though one of its major advantages lies in the fact that the matrices of the governing equation need not be known, in this work the thermophysical properties of the system will be known, so this factor does not come into play. The methodology in the described studies has been applied to systems with a relatively low number of inputs $(1-3)$ and outputs $(2-27)$, while the model considered in this work is on the order of thousands of inputs and outputs due to continuous variation of the heat flux boundary conditions and temperature distribution in space. The complexity associated with the large number of inputs is due to the fact that, in the linear case, an element reduced model (ERM) was required for each input and superposition was used to reconstruct the solution. For the case considered here where each node at the surface of the airfoil represents an input to the system, creation of an ERM for each node is impractial. More fundamentally, while an eigenmode basis will likely provide some means for reduction of the problem, it may not be the optimal basis for capturing the dynamics of the system with the fewest possible number of modes. Consideration of alternative basis representations must therefore be considered.

Within the area of reduced-basis modal methods for heat transfer problems, attempts have been made to augment the eigenmode basis of the thermal system or develop an alternative basis for representation of the system. ${ }^{16-22}$ Shore ${ }^{16}$ utilized an eigenvector basis for nonlinear problems consisting of the eigenvectors based on thermal properties evaluated at the initial temperature conditions and those based on thermal properties evaluated for a temperature distribution corresponding to a nonlinear steady-state problem. To enhance the accuracy and efficiency of the solution, the eigenvector basis was augmented with additional adaptive vectors and analytically generated vectors. Large reductions in size were achieved for conduction-dominated problems with simple geometry and boundary conditions. For problems with complex spatial and temporal variation in heating, additional effort was employed to generate alternative basis vectors. As opposed to an eigenvector basis, Nour-Omid ${ }^{22}$ utilized a Lanczos vector basis for reduction of the transient heat transfer problem. The advantage of such an approach is that the vectors can be generated relatively inexpensively, can produce more accurate solutions than those obtained with an eigenvector basis, and can lead to a reduced system in tridiagonal form for a more computationally efficient solution. An adaptation of this methodology was developed by Cardona and Idelsohn ${ }^{21}$ who obtained the first basis vector as the system response for the first time step and then introduced this vector into the Lanczos algorithm. The authors also extended the method and solved nonlinear thermal problems by introducing new basis vectors which are derivatives of the pre-existing basis vectors with respect to their own amplitude parameters. Another work ${ }^{20}$ discussed the selection of a starting vector to the Lanczos algorithm which is related to the time variation of the heat supply vector.

Another method that has been applied to thermal and structural dynamics problems and gives a higherorder modal solution to the problem is known as the force-derivative method. ${ }^{17-19,23}$ This approach is advantageous in that these higher-order solutions converge to an accurate response using fewer modes than lower-order methods such as the mode-displacement or mode-acceleration methods. Its derivation results from successive integration by parts of the convolution integral form of the solution and results in terms which are related to the forcing function and its time derivatives. The resulting additional terms offer an improved approximation of the higher modes which would otherwise be neglected in a standard mode-displacement approach. The first-order force-displacement relation can be recognized as the mode-acceleration method with one correction term that depends on the forcing function. This method was applied with success to linear transient thermal systems ${ }^{18,19}$ and was later extended for application to nonlinear transient thermal problems. ${ }^{17}$ 
While the above reduced-basis methods provide efficient techniques for reducing the order of transient thermal problems while maintaining sufficient accuracy, they rely largely on the matrices constituting the governing system of equations rather than the actual response of the system to excitations that it will likely see in practice. Methods using an eigenvector basis require solution of a large eigenvalue problem, while augmentation of the eigenvector basis often requires trial and error to capture the correct subspace. Furthermore, the use of such a basis may not be optimal in the sense of capturing the most energy with the fewest number of modes for a given problem. In this work, we will focus on basis representations other than eigenvector space which are derived from the response of the system to typical excitations. If characteristic transient responses of the transient thermal system can be adequately characterized a priori, a basis with established optimality properties can be obtained using POD.

The proper orthogonal decomposition (POD), also known as the Karhunen-Loève decomposition, principal components analysis, singular systems analysis, and singular value decomposition, is a modal technique in which empirical data is processed using statistical methods to obtain models which capture the dominant physics of a system using a finite number of modes. ${ }^{24}$ The fundamental basis for use of POD as a reduced-order modeling method is its ability to represent high-dimensional systems in a low-dimensional, approximate manner while still maintaining a high degree of accuracy. The choice of the POD basis as opposed to any other basis such as an eigenvector basis is due to its optimality condition of providing the most efficient way of capturing the dominant modes of a system with a finite number of modes. ${ }^{25}$ In addition to heat transfer problems, the POD has been used in numerous applications such as turbulence, ${ }^{26}$ structural dynamics ${ }^{27,28}$ aerodynamics, ${ }^{29}$ and control theory ${ }^{30}$ among others.

The use of POD for thermal problems is prevalent in the literature. In two relevant works, the method was applied to a linear transient thermal system in which the governing equations were decoupled in order to solve them analytically over time. ${ }^{31,32}$ Application of the method to cases with time-independent and time-varying loading showed relative errors of less than $1 \%$ and less than $3 \%$, respectively. ${ }^{31}$ In a related work ${ }^{33}$ POD was applied to a nonlinear transient thermal system and a technique was developed for updating the system matrices in cases where the thermal conductivity must be recomputed at every time step. This strategy involved separating the solution-dependent part of the element matrix from the geometry-dependent part to reduce computation times. Application of the methodology to systems with nonlinearity due to temperaturedependent material properties showed average relative error of less than $1 \%$ with respect to the full-order model. Furthermore, it was shown that for a certain range of degrees of freedom, significant improvement in computation time can be achieved using the methodology outlined in Ref. 33. In another study, ${ }^{34}$ an approach was developed to incorporate an understanding of the input operator to the system. A low-order model was developed using POD in which an external control input is included by separating the external stimuli from the ordinary differential equations resulting from projection onto the reduced subspace.

In addition to its use in solving forward heat transfer problems, POD has also been applied in solution of nonlinear inverse heat transfer problems. One example is its use in estimating the time-varying strength of a heat source in a two-dimensional system. ${ }^{35}$ The system consisted of a square domain with a time-varying heat source at a known location. Thermal conductivity was taken to be a strong function of temperature thus resulting in nonlinear governing equations. Drastic reduction in the number of degrees of freedom was achieved using the proposed method while maintaining solution accuracy of the same level as that of a traditional method. A subsequent work utilized POD in the solution of nonlinear inverse natural convection problems. ${ }^{36}$ The goal was to estimate the time-varying strength of a heat source while reducing the order of the computations involved. The reduced-order solution was compared with that obtained using the traditional method of employing the Boussinesq equation. The reduced-order method was shown to accurately reproduce the results obtained using the traditional method for various shapes of the heat source function at reduced computational cost. A later work ${ }^{37}$ applied POD to the inverse problem of estimating the unknown thermal conductivity and convective heat transfer coefficient of a system. The modal coordinates of the basis vectors were allowed to be a nonlinear function of the retrieved parameters. Results showed that the method was robust and numerically stabilizing while also exhibiting favorable regularization due to the ability of POD to filter out high frequency error.

While the above works represent significant progress in the area of reduced-order thermal modeling, this work more specifically seeks to examine the robustness of the POD for thermal solution within a hypersonic vehicle aerothermoelastic framework. The goal is to be able to generate the POD basis a priori, and then use this basis throughout the aerothermoelastic simulation. However, the problem is complicated due to the fact that the thermal boundary conditions are dependent on aerodynamic flow parameters, which are 
effected by the structural dynamic response. Thus, the ability to characterize the space which the basis vectors must span a priori must be investigated. Additionally, it is desirable that the same basis can be used in simulations with varying flight conditions such as Mach number, altitude, and angle-of-attack. Thus, the robustness of the basis to changes in these parameters must be considered. Further complicating the problem are the fact that the boundary conditions are time-dependent and nonlinear due to radiation and temperature-dependent convection.

A previous work by the authors ${ }^{38}$ provided progress toward reduction of the transient heat transfer problem, however there are limitations associated with that formulation. Note that all of the results shown in that work were for cases with heat flux boundary conditions that were constant in time. This work will extend the reduced-order thermal formulation of Ref. 38 to examine the ability to handle the time-dependence of the heat flux boundary condition. An overview of POD will be given and the optimality conditions of the basis will be described. The method for basis generation will be outlined along with the two methods used to solve the governing system of ordinary differential equations for the modal coordinates. A representative control surface structure to be used in the examples will then be described. Numerical examples will be carried out for varying boundary conditions and results will be presented to demonstrate the validity of the approach.

\section{Thermal POD Formulation}

\section{II.A. Creation of POD Basis}

The transient heat transfer problem is solved in modal space with modes from POD analysis due to the optimality properties of the basis. ${ }^{31}$ The method of snapshots ${ }^{39}$ is used for determination of the POD basis vectors. In this case, the snapshots are defined as vectors of nodal temperatures at various time instants and are computed from high-fidelity finite element analysis. The goal of the POD formulation is to express the vector of nodal temperatures, $T$, at any time instant as a linear combination of the basis, $\varphi(x, y, z)$, with coefficients $c(t)$, i.e.,

$$
\left\{\begin{array}{c}
T_{1} \\
\vdots \\
T_{s}
\end{array}\right\}=c_{1}(t)\left\{\begin{array}{c}
\varphi_{1}^{(1)} \\
\vdots \\
\varphi_{s}^{(1)}
\end{array}\right\}+c_{2}(t)\left\{\begin{array}{c}
\varphi_{1}^{(2)} \\
\vdots \\
\varphi_{s}^{(2)}
\end{array}\right\}+\cdots+c_{r}(t)\left\{\begin{array}{c}
\varphi_{1}^{(r)} \\
\vdots \\
\varphi_{s}^{(r)}
\end{array}\right\},
$$

where $s$ is the total number of degrees of freedom in the finite element model and $r$ is the total number of POD basis vectors retained after truncation. The basis is computed by first generating the snapshot matrix, $A$, given by,

$$
A=\left[\begin{array}{cccc}
T_{1}^{(1)} & T_{1}^{(2)} & \ldots & T_{1}^{(n)} \\
T_{2}^{(1)} & T_{2}^{(2)} & \ldots & T_{2}^{(n)} \\
\vdots & \vdots & \ddots & \vdots \\
T_{s}^{(1)} & T_{s}^{(2)} & \ldots & T_{s}^{(n)}
\end{array}\right]=\left[a_{1}, a_{2}, \ldots, a_{n}\right]
$$

where $T_{i}^{(j)}$ indicates the $i$-th entry of the $j$-th snapshot, $n$ is the number of snapshots taken and $a_{j}$ refers to column vector corresponding to the $j$-th snapshot. The correlation matrix, $C$, is then found, whose entries are the inner products of the corresponding snapshots, given by

$$
C_{i j}=\frac{1}{n} a_{i}^{T} a_{j} \text { or } C=\frac{1}{n} A^{T} A .
$$

Solution of the eigenvalue problem,

$$
C v_{i}=\lambda_{i} v_{i},
$$

yields the eigenvectors and eigenvalues of the correlation matrix where $v_{i}$ with $\left\|v_{i}\right\|=1$ indicates the $i$-th eigenvector of $C$ corresponding to the $i$-th largest eigenvalue of $C$. Note that the eigenvectors are sorted such that they correspond with eigenvalues that are sorted in decreasing magnitude so that the POD basis vectors will be sorted in order of decreasing energy. The eigenvalues, $\lambda$, and eigenvectors, $v$, of the correlation matrix are then used to generate the POD basis vectors, $\varphi$, which are expressed as a linear combination of 
the snapshots, i.e.,

$$
\varphi_{k}=\frac{1}{\sqrt{n \lambda_{k}}} \sum_{i=1}^{n} v_{i}^{(k)} a_{i}=\frac{1}{\sqrt{n \lambda_{k}}} A v_{k}
$$

where $v_{i}^{(k)}$ is the $i$-th entry of the $k$-th eigenvector. The POD modal matrix, $\Phi$, can then be assembled with the POD basis vectors, $\varphi_{i}$, stored as columns of the matrix. The POD basis is then truncated to a reduced set of POD vectors, $\bar{\Phi}$, thus leading to a reduction in the number of degrees of freedom in the problem. Note that both the full and truncated POD sets are orthogonal, i.e.

$$
\begin{gathered}
\Phi^{T} \Phi=I_{n} \\
\bar{\Phi}^{T} \bar{\Phi}=I_{r},
\end{gathered}
$$

where $I_{n}$ represents the identity matrix of dimension $n$ and $I_{r}$ refers the the identity matrix of dimension $r$.

The columns of the snapshot matrix can be expressed as linear combinations of the basis vectors with coefficients using the expression ${ }^{31}$

$$
A=\Phi \eta,
$$

where $\eta$ is a square matrix of coefficients of the full set of POD basis vectors and can be calculated by making use of the orthogonality of the POD basis as

$$
\eta=\Phi^{T} A
$$

As the basis will be truncated to reduce the problem, the snapshot matrix can at best be approximated via a linear combination of the truncated POD modal matrix, $\bar{\Phi}$, given by

$$
A \approx \bar{\Phi} \bar{\eta}
$$

where $\bar{\eta}$ is a square matrix of coefficients of the truncated set of POD basis vectors and can be calculated in a manner similar to that of Eq. (8) using

$$
\bar{\eta}=\bar{\Phi}^{T} A,
$$

where the orthogonality of $\bar{\Phi}$ is utilized. As the truncated basis can only approximate the snapshots, the goal is to find the optimal basis such that the approximated snapshot matrix, $\bar{\Phi} \bar{\eta}$, represents as closely as possible the actual snapshot matrix, $\Phi \eta$. Let $\Psi$ represent an arbitrary orthonormal $s \times n$ basis and $\bar{\Psi}$ represent the corresponding $s \times r$ truncated basis. Let the error incurred as a result of basis truncation, $\varepsilon$, be given by 31,40

$$
\varepsilon=\|A-\bar{\Psi} \bar{\eta}\|^{2} .
$$

Using Eq. (10) in Eq. (11) and substituting the truncated arbitrary basis, $\bar{\Psi}$, for the truncated POD basis, $\bar{\Phi}$, the error expression becomes ${ }^{31}$

$$
\varepsilon=\left\|A-\bar{\Psi} \bar{\Psi}^{T} A\right\|^{2} .
$$

At this point, the quantity $\bar{\Psi} \bar{\Psi}^{T}$ is recognized as the orthogonal projector that projects onto the $r$-dimensional subspace spanned by the basis, $\bar{\Psi}{ }^{40,41}$ Thus, Eq. (12) can be written as

$$
\varepsilon=\left\|A-P_{\bar{\Psi}, r} A\right\|^{2},
$$

where $P_{\bar{\Psi}, r}$ is the orthogonal projector onto the $r$-dimensional subspace. The error due to basis truncation can then be interpreted as the amount by which the projection of the snapshots onto the truncated basis differs from the snapshots themselves. The objective is therefore to find an orthonormal basis such that for a specified error, $\varepsilon$, a minimum number of columns of the set of basis vectors must be retained. A supplementary condition following from Eq. (7) is that the basis is a linear combination of the snapshots as seen in Eq. (5). It has been shown ${ }^{25}$ that along with this supplementary condition, the minimum error in Eq. (13) occurs when the basis, $\bar{\Psi}$, is chosen to be the POD basis, $\bar{\Phi}$, as given in Eq. (5). Thus the POD basis is optimal with respect to any other linear modal representation in that the first $k$ POD modes contain more energy than the first $k$ modes of any other basis. ${ }^{24}$

An alternative method for calculating the POD basis involves the singular value decomposition (SVD). The full SVD of the $s \times n$ snapshot matrix, $A$, is given by ${ }^{42}$

$$
A=U \Sigma V^{T}
$$


where $U$ is a $s \times s$ orthogonal matrix, $V$ is an $n \times n$ orthogonal matrix, and $\Sigma$ is a $s \times n$ diagonal matrix with

$$
\sigma_{i j}=\left\{\begin{array}{ll}
0 & \text { for } i \neq j \\
\sigma_{i} \geq 0 & \text { for } i=j
\end{array} .\right.
$$

The diagonal entries of $\Sigma$ are known as the singular values of $A$ and are ordered in decreasing order such that $\sigma_{1} \geq \sigma_{2} \geq \ldots \geq 0$. The columns of $U$ and $V$ are the left and right singular vectors of $A$, respectively. The correspondence between the POD basis as derived using the eigenvalues of the correlation matrix, $\lambda_{i}$, and that derived using the SVD can be shown by relating $\lambda_{i}$ to $\sigma_{i}$. Performing the SVD of $C$ in terms of the SVD of $A$, we have

$$
C=\frac{1}{n} A^{T} A=\frac{1}{n}\left(U \Sigma V^{T}\right)^{T} U \Sigma V^{T}=\frac{1}{n} V \Sigma^{T} U^{T} U \Sigma V^{T} .
$$

Taking advantage of the orthogonality of $U$, Eq. (16) becomes

$$
C=\frac{1}{n} V\left(\Sigma^{T} \Sigma\right) V^{T}
$$

From Eq. (17) we can see that $V$ is the matrix of eigenvectors of $C$ and the singular values of $A$ are related to the eigenvalues of $C$ according to ${ }^{40}$

$$
\sigma_{i}^{2}=n \lambda_{i} .
$$

The POD basis vectors can be shown to correspond to the left singular vectors of the snapshot matrix, ${ }^{40}$ if we re-write Eq. (5) in terms of the SVD of $A$, i.e.,

$$
\varphi_{k}=\frac{1}{\sqrt{n \lambda_{k}}} U \Sigma V^{T} v_{k}
$$

Due to orthogonality of $V$, Eq. (19) simplifies to

$$
\varphi_{k}=\frac{1}{\sqrt{n \lambda_{k}}} u_{k} \sigma_{k}
$$

where $u_{k}$ is the $k$-th left singular vector of $A$. Utilizing Eq. (18), we have $\varphi_{k}=u_{k}$. The connection between POD and SVD allows for justification of the claim of basis optimality due to the ability of the SVD to provide an optimal low-rank approximation to a matrix. Consider an approximation to $A$ written as a partial sum of rank-one matrices formed from the outer product of the left and right singular vectors with the singular value as the scalar coefficient, given by

$$
A=\sum_{j=1}^{r} \sigma_{j} u_{j} v_{j}^{T}
$$

where $r$ corresponds to the number of POD basis vectors retained after truncation. It can be shown that the $r$-th partial sum captures the maximum possible amount of energy of $A$, where energy is defined in either the 2-norm or Frobenius norm sense. ${ }^{41-43}$ Alternatively stated, no other rank $r$ matrix can be closer to $A$ in the 2-norm or in the Frobenius norm. To examine the connection between this optimality property of the SVD and the optimality of the POD basis, consider Eq. (11) written in terms of the truncated set of left singular vectors, $\bar{U}$, corresponding to the truncated set of POD basis vectors, $\bar{\Phi}$, given by

$$
\varepsilon=\left\|A-\bar{U} \bar{U}^{T} A\right\|^{2} .
$$

Now, expressing $A$ in terms of its full SVD leads to,

$$
\varepsilon=\left\|U \Sigma V^{T}-\bar{U} \bar{U}^{T} U \Sigma V^{T}\right\|^{2} .
$$

At this point, the quantity $\bar{U} \bar{U}^{T} U$ is recognized as the projection of $U$ onto the space spanned by $\bar{U}$ and performs the action of zeroing the columns of $U$ that correspond to the excluded POD basis vectors. Thus, Eq. (23) becomes

$$
\varepsilon=\left\|\sum_{j=1}^{n} \sigma_{j} u_{j} v_{j}^{T}-\sum_{j=1}^{r} \sigma_{j} u_{j} v_{j}^{T}\right\|^{2},
$$


and the optimality of the POD basis is demonstrated due to the optimality property of the SVD described above. The absolute error associated with the $r$-dimensional POD subspace is associated with the eigenvalues of the correlation matrix, $C$, and the singular values of the snapshot matrix, $A$, and is given by ${ }^{40}$

$$
\varepsilon=\sum_{j=r+1}^{n} \sigma_{j}^{2}=n \sum_{j=r+1}^{n} \lambda_{j}
$$

where $\varepsilon$ is defined in the Frobenius norm sense. A relative error tolerance, $\varepsilon_{r e l}$, can be defined such that if the relative error is to be less than an error tolerance, $\varepsilon_{T}$, i.e.,

$$
\frac{\varepsilon}{\|A\|^{2}}=\varepsilon_{r e l} \leq \varepsilon_{T}
$$

the number of basis vectors retained, $r$, should be the smallest integer that

$$
\frac{\sum_{j=1}^{r} \sigma_{j}^{2}}{\sum_{j=1}^{n} \sigma_{j}^{2}}=\frac{\sum_{j=1}^{r} \lambda_{j}}{\sum_{j=1}^{n} \lambda_{j}} \geq 1-\varepsilon_{T} .
$$

Note that the left-hand side of Eq. (27) can be interpreted as the energy of the included basis vectors relative to the energy of the full set of basis vectors. Furthermore, $\varepsilon_{r e l}$ can be interpreted as the energy of the excluded basis vectors relative to the energy of the full set of basis vectors, given by

$$
\varepsilon_{r e l}=\frac{\varepsilon}{\|A\|^{2}}=\frac{\sum_{j=r+1}^{n} \sigma_{j}^{2}}{\sum_{j=1}^{n} \sigma_{j}^{2}}=\frac{\sum_{j=r+1}^{n} \lambda_{j}}{\sum_{j=1}^{n} \lambda_{j}} .
$$

Thus, the magnitude of each of the eigenvalues of the correlation matrix can be used in determining the number of POD basis vectors that can be removed from the set.

\section{II.B. Solution of System for Modal Coordinates}

\section{II.B.1. Decoupled System: Analytical Solution}

One method employed in this work to solve the system of ordinary differential equations for the modal coordinates of the POD basis vectors involves decoupling the equations and solving each analytically. The advantage of the analytical solution is that it avoids the need to time-march the solution and allows for direct generation of the temperature distribution at any time instant of interest. Additionally, it eliminates any error incurred due to numerical integration. The analytical solution is also useful in assessing the accuracy of approximate numerical solutions for linear cases in which the load vector is a known function of time due to its ability to provide an exact solution. The procedure begins with the original system of transient thermal finite element equations given by

$$
M \dot{T}+K T=F(t),
$$

where $M$ is the thermal capacitance matrix, $K$ is the thermal conductivity matrix, and $F$ is the thermal load vector. Note that at this stage we assume that both $K$ and $M$ are not diagonal. As the set of equations given in Eq. (29) is first order in time, we must also specify a vector of initial temperatures,

$$
T(t=0)=T_{0} .
$$

Because the initial condition must be specified for each equation in the analytical solution, it is convenient to work with a homogeneous initial condition. Thus we define a new temperature variable given by $\bar{T}=T-T_{0} \cdot{ }^{31}$ This transformation results in transforming Eq. (29) such that it becomes

$$
M \dot{\bar{T}}+K \bar{T}=G(t),
$$

where $G(t)=F(t)+K T_{0}$. The next step is to transform the system from physical space to modal space, thus reducing from an $s$ by $s$ system to an $r$ by $r$ system. This is accomplished by first expressing the temperature vector as a linear combination of the truncated set of POD basis vectors, $\bar{T}=\bar{\Phi} c$, and then premultiplying the equation by $\bar{\Phi}^{T}$ where $(\cdot)^{T}$ indicates the transpose of a matrix. The system of equations is then of the form

$$
\bar{\Phi}^{T} M \bar{\Phi} \dot{c}+\bar{\Phi}^{T} K \bar{\Phi} c=\bar{\Phi}^{T} G .
$$


The generalized thermal capacitance matrix, $m$, generalized thermal conductivity matrix, $k$, and generalized load vector, $g$ are then identified such that

$$
\begin{aligned}
m & =\bar{\Phi}^{T} M \bar{\Phi} \\
k & =\bar{\Phi}^{T} K \bar{\Phi} \\
g & =\bar{\Phi}^{T} G .
\end{aligned}
$$

Note that because the POD basis vectors are not eigenvectors of the generalized eigenvalue problem, $m$ and $k$ will still not be diagonal at this stage. Thus, we now solve the generalized eigenvalue problem given by

$$
\left(k-\gamma_{i} m\right) w_{i}=0,
$$

where $\gamma_{i}$ is the $i$-th eigenvalue and $w_{i}$ is the corresponding $i$-th eigenvector. Note that this step is intentionally performed after transformation to modal space so that the size of the eigenvalue problem to be solved is minimized. After assembling the eigenvectors $w_{i}$ as columns of the modal matrix $W$, we now express $c$ as a linear combination of the eigenvectors of the generalized eigenvalue problem so that $c=W \tilde{c}$. The system of equations is then premultiplied by $W^{T}$ such that the system becomes

$$
W^{T} m W \dot{\tilde{c}}+W^{T} k W \tilde{c}=W^{T} g .
$$

The transformed generalized thermal capacitance matrix, $\tilde{m}$, transformed generalized thermal conductivity matrix, $\tilde{k}$, and transformed generalized load vector, $\tilde{g}$ are then identified such that

$$
\begin{aligned}
\tilde{m} & =W^{T} m W \\
\tilde{k} & =W^{T} k W \\
\tilde{g} & =W^{T} g .
\end{aligned}
$$

Due to orthogonality of the eigenvectors with respect to $m$ and $k, \tilde{m}$ and $\tilde{k}$ will be diagonal matrices, thus decoupling the system of ordinary differential equations. Furthermore, by enforcing the eigenvectors, $W$, to be normalized with respect to $m, \tilde{m}$ is reduced to the identity matrix and the $i$-th equation of the system can be expressed as

$$
\dot{\tilde{c}}_{i}+\tilde{k}_{i} \tilde{c}_{i}=\tilde{g}_{i},
$$

where $\tilde{k}_{i}$ is the $i$-th diagonal entry of $\tilde{k}$. Using the fact that the initial condition was made to be homogeneous, the solution of the $i$-th equation for an arbitrary time-dependent generalized load, $\tilde{g}(t)$, is given by

$$
\tilde{c}_{i}(t)=\mathrm{e}^{-\tilde{k}_{i} t} \int_{0}^{t} \tilde{g}_{i}(\tau) \mathrm{e}^{\tilde{k}_{i} \tau} d \tau, \quad i=1, \ldots, r,
$$

where $\tau$ is a dummy variable of integration and $t$ is the time instant of interest. For a generalized load that is independent of time, the solution is given by

$$
\tilde{c}_{i}(t)=\tilde{g}_{i} \frac{1-\mathrm{e}^{-\tilde{k}_{i} t}}{\tilde{k}_{i}}, \quad i=1, \ldots, r .
$$

Once all of the transformed modal coordinates have been calculated, the sequence of transformations can be reversed to obtain the physical temperatures using

$$
T(t)=\bar{\Phi} W \tilde{c}(t)+T_{0} .
$$

Note that for the linear case, the matrix product $\bar{\Phi} W$ must only be evaluated once, and solution of the transient thermal problem is reduced to a matrix-vector product and a vector sum. An overview of the sequence of equation transformations is given in Fig. 3. 


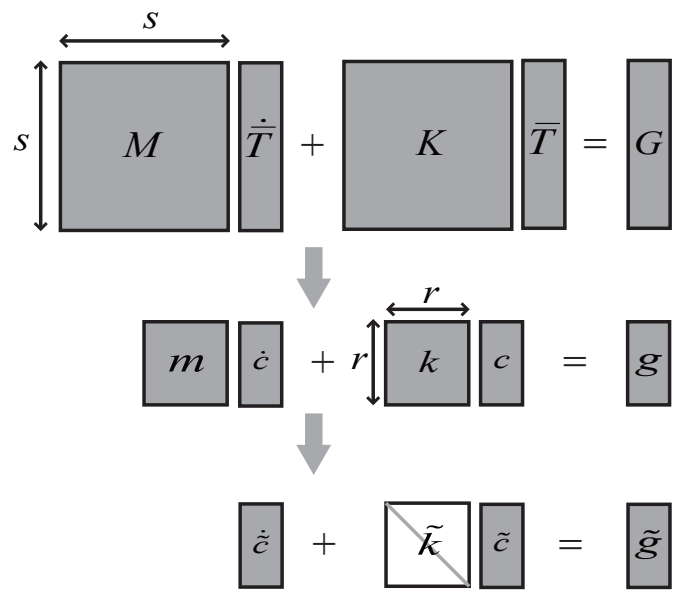

Figure 3. Sequence of transformations of heat transfer equations from full system to decoupled reduced-order system.

\section{II.B.2. Coupled System: Numerical Integration}

In addition to decoupling the system and solving the equations analytically, results were also obtained from numerical solutions of the coupled system. Numerical solutions of the coupled thermal problem may prove to be more useful than decoupled analytical solutions within hypersonic vehicle aerothermoelastic simulation frameworks. This is due to the fact that the heat flux depends on various factors such as deformation and aerodynamic flow properties that are not known ahead of time. The thermal load vector must therefore be sampled at specific time instants and integrated over time numerically, thus the analytical solution loses its utility in these cases. Note that one could perform the integral in Eq. (38) numerically and still use the decoupled solution method. However at that point it may be more computationally efficient to integrate the equations numerically and avoid diagonalizing the system matrices. Solving the coupled system directly avoids the need to solve an eigenvalue problem to decouple the equations. This is especially important for nonlinear problems in which the thermal capacitance matrix and thermal conductivity matrix change with temperature and an eigenvalue problem would need to be solved at every time step if the equations were to be decoupled.

For the numerical solution, the transient equations are solved using a numerical time-marching algorithm. ${ }^{44,45}$ For nonlinear cases in which the system matrices or load vector depend on the temperatures at the current time step, Newton-Raphson iterations can be employed at each time step. To begin the formulation, consider a general system of first order coupled ordinary differential equations of the form

$$
M \dot{T}+K T=F(t),
$$

The first step will be to reduce the system and transform from physical space to modal space using Eq. (33). For the numerical solution, we will not perform the additional step to solve the eigenvalue problem and decouple the equations. Rather, the reduced-order coupled system will be integrated numerically at this stage. We denote a time instant in the response of the system by $t_{n}$ such that the time instant at the next time step is given by by $t_{n+1}=t_{n}+\Delta t$, where $n=0,1,2, \ldots, N$. A parameter $\theta$ is introduced to represent the response of the system at an intermediate time, $t_{\theta}$, such that $t_{\theta}=t_{n}+\theta \Delta t$, where $0 \leq \theta \leq 1$. Expressing Eq. (41) at time $t_{\theta}$ in modal space, we have,

$$
m \dot{c}_{\theta}+k c_{\theta}=g\left(t_{\theta}\right),
$$

where the subscript $\theta$ indicates the vector of unknowns, at time instant $t_{\theta}$. We now introduce approximations to $c$, its time-derivative, and $g$ at $t_{\theta}$ given by

$$
\begin{aligned}
\dot{c}_{\theta} & =\frac{c_{n+1}-c_{n}}{\Delta t} \\
c_{\theta} & =(1-\theta) c_{n}+\theta c_{n+1} \\
g\left(t_{\theta}\right) & =(1-\theta) g_{n}+\theta g_{n+1} .
\end{aligned}
$$


Substituting Eqs. (43) into Eq. (42) leads to the recurrence relation given by ${ }^{44}$

$$
\left[\theta k+\frac{1}{\Delta t} m\right] c_{n+1}=\left[-(1-\theta) k+\frac{1}{\Delta t} m\right] c_{n}+(1-\theta) g_{n}+\theta g_{n+1},
$$

where $c_{n+1}$ is the unknown to be found and $c_{n}$ is the solution from the previous time step. The recurrence relation given in Eq. (44) is of the form

$$
L c_{n+1}=b_{n+1},
$$

where

$$
\begin{aligned}
L & =\theta k+\frac{1}{\Delta t} m \\
b_{n+1} & =\left[-(1-\theta) k+\frac{1}{\Delta t} m\right] c_{n}+(1-\theta) g_{n}+\theta g_{n+1} .
\end{aligned}
$$

Equation (44) represents a family of recurrence relations in which the particular numerical scheme depends on the chosen value of $\theta$. For $\theta=0, \frac{1}{2}, \frac{2}{3}$, and 1, the algorithm represents the forward Euler, Crank-Nicolson, Galerkin, and backward Euler method, respectively. Additionally, if $\theta=0$, the algorithm is explicit, while for $\theta>0$, the algorithm is implicit. The requirements for convergence of the selected numerical scheme are given by the Lax Equivalence Theorem which states that for a well-posed initial value problem and a consistent discretization scheme, stability is the necessary and sufficient condition for convergence. ${ }^{42,46}$ The family of $\theta$ algorithms described above is unconditionally stable in the linear case for $\theta \geq \frac{1}{2}$ while an extension for nonlinear systems ${ }^{47}$ was also shown to be unconditionally stable for $\theta \geq \frac{1}{2}$. For $\theta<\frac{1}{2}$, the algorithm is conditionally stable and the eigenvalue stability region is such that the critical time step is given by

$$
\Delta t_{c r}=\frac{2}{1-2 \theta} \frac{1}{\gamma_{m}}
$$

where $\gamma_{m}$ is the largest system eigenvalue. For this work, the Crank-Nicolson scheme corresponding to $\theta=\frac{1}{2}$ is used due to its second-order accuracy.

Since the equations are solved in modal space and the initial temperatures are known in physical space, the initial values of the modal coordinates must first be calculated before time-marching can proceed. Using the transformation between physical space and modal space, we begin by expressing the vector of initial temperatures in physical space as can be expressed as

$$
T_{0}=\bar{\Phi} c_{0},
$$

where $c_{0}$ is the vector of initial values of the modal coordinates. Expanding this expression in terms of the individual POD vectors, Eq. (48) becomes

$$
T_{0}=c_{0}^{(1)} \varphi_{1}+c_{0}^{(2)} \varphi_{2}+\cdots+c_{0}^{(r)} \varphi_{r}
$$

where $\varphi_{i}$ indicates the $i$-th basis vector and $c_{0}^{(i)}$ refers to the corresponding $i$-th modal coordinate at time $t_{0}$. To find the initial value of the $i$-th modal coordinate, Eq. (49) is premultiplied by the transpose of the $i$-th basis vector, $\varphi_{i}^{T}$, to obtain

$$
\varphi_{i}^{T} T_{0}=c_{0}^{(1)} \varphi_{i}^{T} \varphi_{1}+c_{0}^{(2)} \varphi_{i}^{T} \varphi_{2}+\cdots+c_{0}^{(r)} \varphi_{i}^{T} \varphi_{r}
$$

Recall that the basis vectors are an orthonormal set such that

$$
\varphi_{i}^{T} \varphi_{j}=\delta_{i j}
$$

where $\delta_{i j}$ is the Kronecker delta. Thus, the right-hand side of Eq. (50) reduces to $c_{0}^{(i)}$ and the left-hand side gives its value. By premultiplying Eq. (49) by each of the basis vectors, the complete vector of initial modal coordinates can be found and time-marching of the system can proceed. 


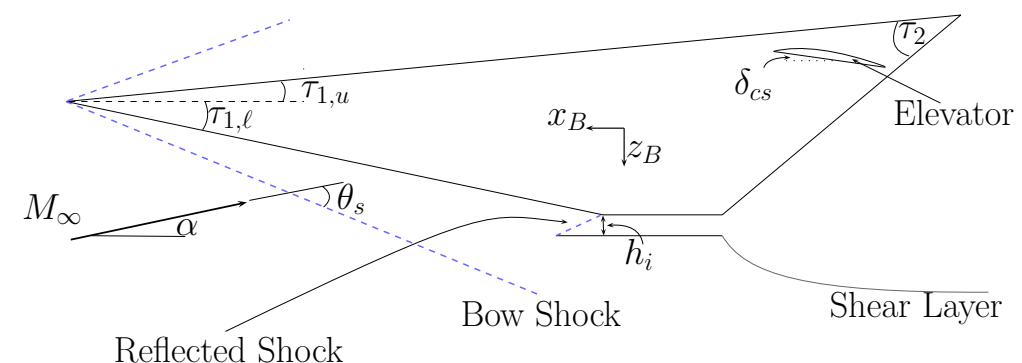

Figure 4. Overall HSV geometry illustrating position of control surface.

\section{Control Surface Model}

The methodology developed in this work is applied to a representative control surface structure as the control surfaces are expected to have a strong contribution to the dynamics of the vehicle. The hypersonic vehicle configuration considered in this study, as developed in a previous work, ${ }^{48}$ is shown in Fig. 4 . A finite element model of a representative hypersonic vehicle elevator has been created for use in this study. The airfoil cross-section is that of a double wedge with a maximum thickness of $4 \%$ chord length. ${ }^{49-51}$ The chord length at the root is $5.2 \mathrm{~m}(17 \mathrm{ft})^{48}$ and the leading edge is swept by $34^{\circ}$ while the trailing edge is swept by $18^{\circ} .{ }^{52}$ Planform and cross-sectional views of the airfoil are given in Fig. 5 and Fig. 6, respectively.

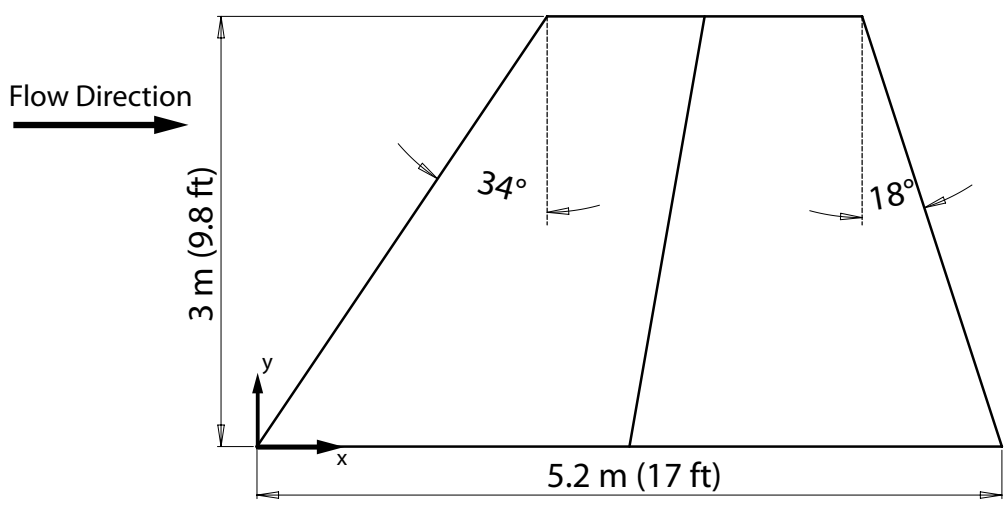

Figure 5. Planform geometry of control surface model.

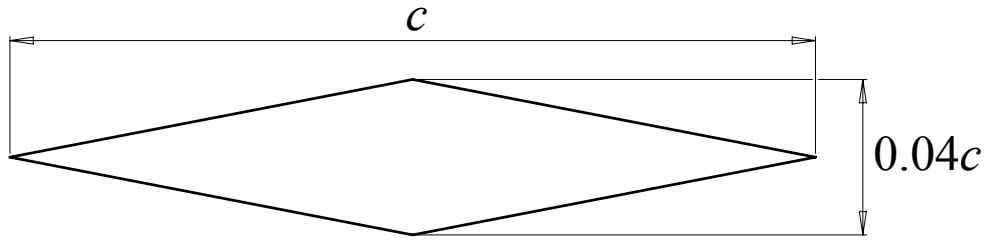

Figure 6. Cross-sectional geometry of control surface model.

Due to the severe aerodynamic heating experienced in hypersonic flight, layers of thermal protection material are needed to keep the temperature of the structure below maximum temperature limits. A survey of the literature revealed a wide range of design strategies for mitigating the high temperatures experienced in hypersonic flight. ${ }^{53-60}$ This study considers a thermal protection system consisting of an outer heat shield and middle insulation layer on top of the structure as shown in Fig. 7. The material for the heat shield was chosen to be René 41 as it was found to be efficient in terms of mechanical properties at elevated temperatures. ${ }^{59}$ For the insulation layer, three different materials were considered in the preliminary materials evaluation: Internal Multiscreen Insulation (IMI) ${ }^{54}$ High Temperature Flexible Min-K, ${ }^{59}$ and Q-Fiber Felt. ${ }^{54}$ Of these, the Min-K insulation, which is a proprietary silica based material faced with Astroquartz cloth, ${ }^{59}$ was selected 


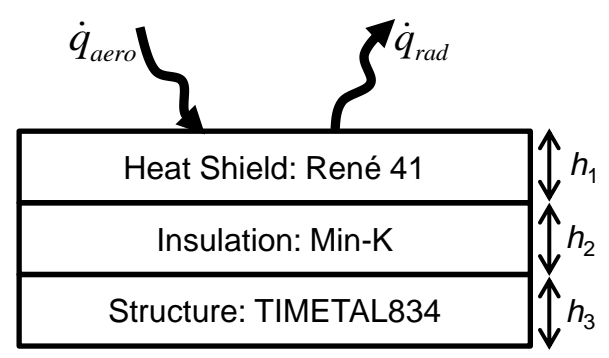

Figure 7. Schematic of material stacking scheme at outer mold line of structure.

due to its relatively low thermal diffusivity. For the structure, the Titanium alloy TIMETAL 834 (formerly known as IMI 834) was chosen. The advantage of using this alloy is its maximum application temperature, $T_{\max }$, of $600^{\circ} \mathrm{C}$ compared with that of Ti-6242S $\left(520^{\circ} \mathrm{C}\right)$, Ti-6242 $\left(450^{\circ} \mathrm{C}\right)$, Ti-811 $\left(400^{\circ} \mathrm{C}\right)$, and Ti-6-4 $\left(300^{\circ} \mathrm{C}\right) .{ }^{61}$ The thermal and mechanical properties of the three materials employed in the model are shown in Table 1, where "T-dep." indicates that the property is temperature-dependent. In addition to those, the emissivity, $\epsilon$, of the heat shield was taken to be $0.85 .^{52,62}$

Table 1. Structural and thermal material properties used in the study. ${ }^{52,59,63-65}$

\begin{tabular}{l||c|c|c|c|c|c|c|c}
\hline \hline & $\begin{array}{c}\rho \\
{\left[\mathrm{kg} / \mathrm{m}^{3}\right]}\end{array}$ & $\begin{array}{c}E \\
{[P a]}\end{array}$ & $\nu$ & $\begin{array}{c}\alpha_{T} \\
{[\mu \mathrm{m} / \mathrm{m} / \mathrm{K}]}\end{array}$ & $\begin{array}{c}k_{T} \\
{[W / \mathrm{m} / \mathrm{K}]}\end{array}$ & $\begin{array}{c}c_{p} \\
{[\mathrm{~J} / \mathrm{kg} / \mathrm{K}]}\end{array}$ & $\begin{array}{c}T_{\max } \\
K\end{array}$ & $\begin{array}{c}h \\
{[\mathrm{~mm}]}\end{array}$ \\
\hline René 41 & 8240 & T-dep. & 0.31 & T-dep. & 18 & 541 & 1500 & 3.8 \\
\hline Min-K & 256 & Neglect & Neglect & Neglect & 0.052 & 858 & 1250 & 3.8 \\
\hline TIMETAL834 & 4550 & T-dep. & 0.31 & 11 & 7 & 525 & 873 & 3.175 \\
\hline \hline
\end{tabular}

The finite element mesh used in the study is shown in Fig. 8. The model contains 2,812 degrees of freedom and 5,580 elements. The heat shield and insulation layer are each modeled using 6-node solid wedge elements while the top and bottom skins and stiffeners are modeled using 3-node, 2-dimensional triangular elements. Of the 5,580 elements in the model, 3,456 are solid elements and 2,124 are triangular elements. The control surface is taken to all-moveable about a hinge line located at the mid-chord ${ }^{48}$ and will thus be connected to the vehicle main body through a torque tube. This attachment is modeled by constraining the region indicated by the black circle in Fig. 8 in all degrees of freedom.

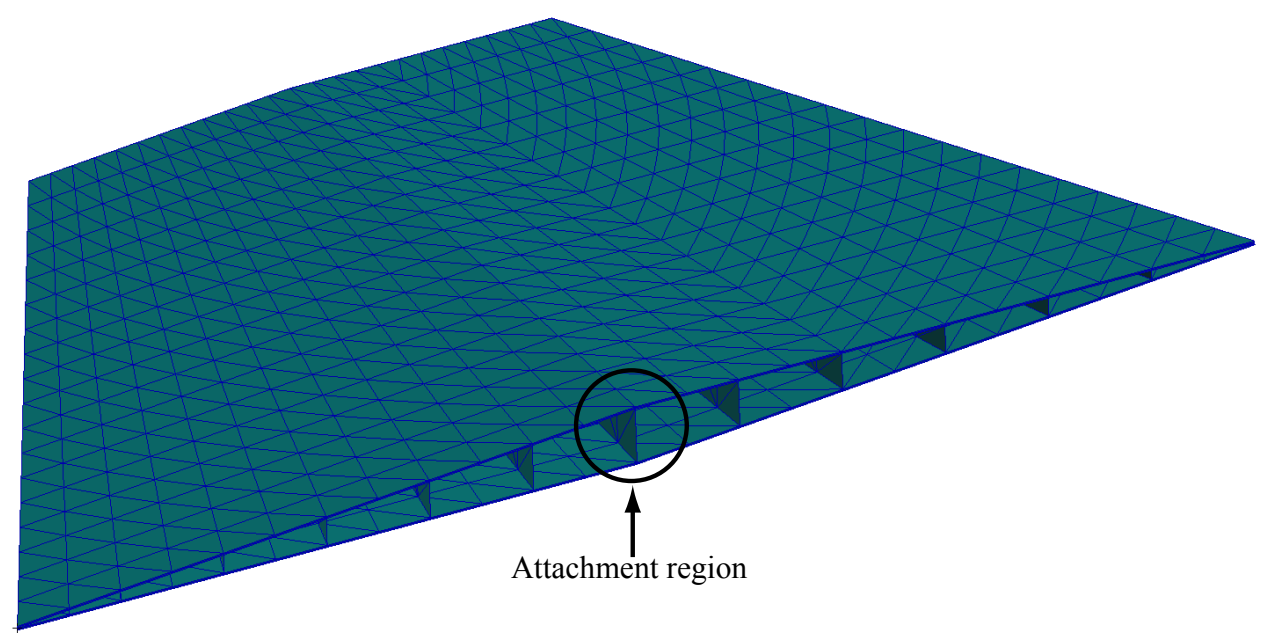

Figure 8. Finite element model of control surface used in study. 


\section{Results}

\section{IV.A. Time-Independent Thermal Load Vector}

The first case that will be considered consists of a uniform heat flux of $10 \mathrm{~W} / \mathrm{cm}^{2}$ that is constant in time and applied at the outer surfaces of the model. The time range considered in this example is $0-200 \mathrm{~s}$. A plot of the temperatures of a selected node at the outer surface of the heat shield, outer surface of the insulation, and skin within the time range considered is given in Fig. 9.

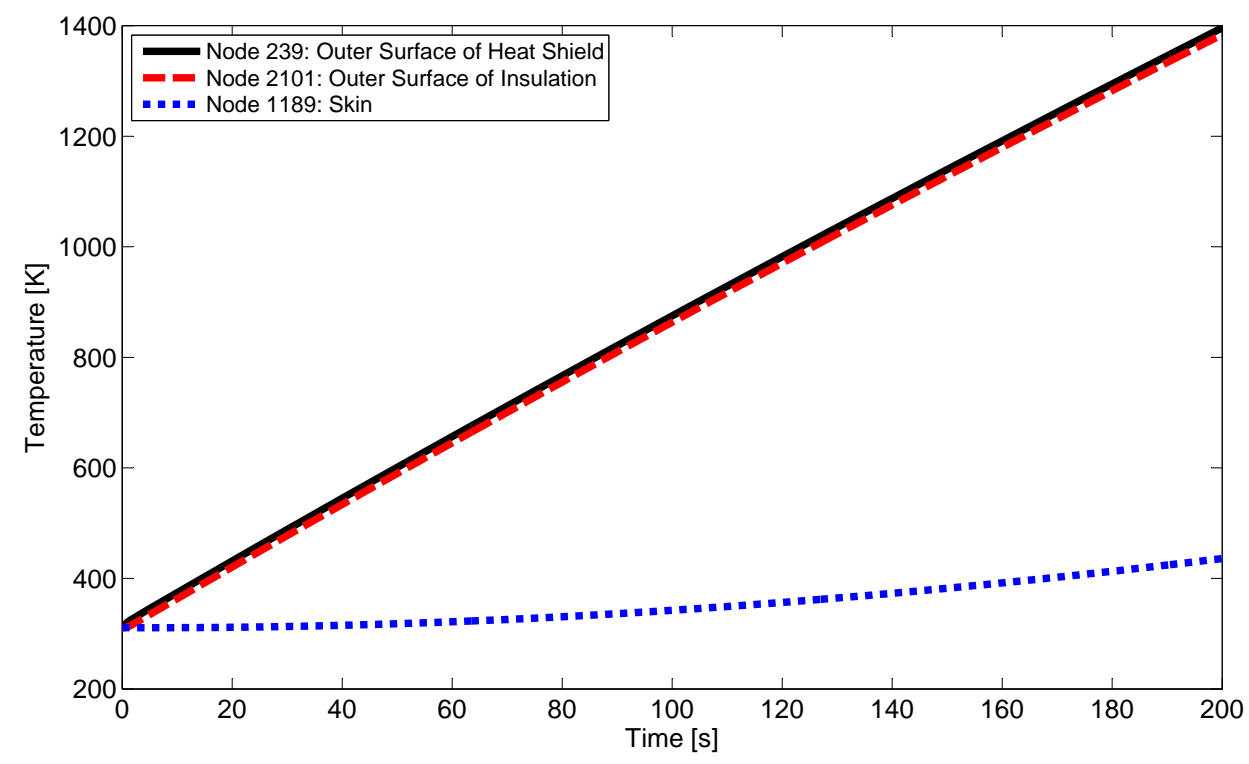

Figure 9. Temperature history of three selected nodes for constant heat flux case.

Note that the temperature decreases significantly between node 2101 and node 1189 due to the effect of the insulation. For the purposes of this study, the high-fidelity finite element solution will be treated as the truth model and error calculations will be made with respect to it. To assess the effect of number of snapshots taken throughout the range of time considered, a high-fidelity transient thermal finite element analysis (FEA) from 0 - $200 \mathrm{~s}$ was carried out using Sol 159 within the finite element code MSC.Nastran. POD bases were then generated based on 5,21, 81, and 401 snapshots of the high-fidelity solution, which correspond to snapshots taken in evenly spaced intervals every 50,10, 2.5, and 0.5 seconds, respectively, between 0 - $200 \mathrm{~s}$. The reduced system was solved using the decoupled analytical approach for all of the results in this section. To quantify the percentage error of the POD solution, a spatial error norm, $e$, is defined such that

$$
e=\sqrt{\sum_{i=1}^{s}\left(\frac{T_{i}^{r}-T_{i}^{f}}{T_{i}^{f}}\right)^{2}} \times 100,
$$

where $T_{i}^{f}$ is the temperature at node $i$ from the full-order solution and $T_{i}^{r}$ is the temperature at node $i$ from the reduced-order solution. Defined in this manner, $e$ can be interpreted as the 2-norm of the vector of percentage errors of the nodes at a given time instant. Results were generated for cases with one, two, and three POD basis vectors retained after truncation. The relative errors as defined in Eq. (28) for the case of 401 snapshots with one, two, and three retained POD basis vectors are given in Table 2. Results

Table 2. Relative energy of excluded modes for cases of one, two, and three retained modes.

\begin{tabular}{c|c}
\hline \hline Number of retained modes & $\varepsilon_{\text {rel }}$ \\
\hline 1 & $3.90 \times 10^{-4}$ \\
\hline 2 & $7.04 \times 10^{-6}$ \\
\hline 3 & $1.22 \times 10^{-9}$ \\
\hline \hline
\end{tabular}


of reduced-order simulations with varying number of retained modes and number of snapshots are given in Fig. 10 with $e$ calculated in 0.5 s intervals.

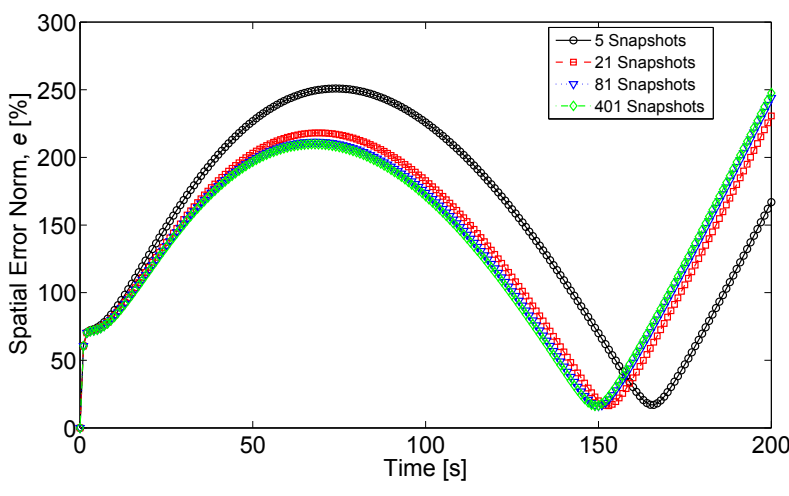

(a) One basis vector retained.

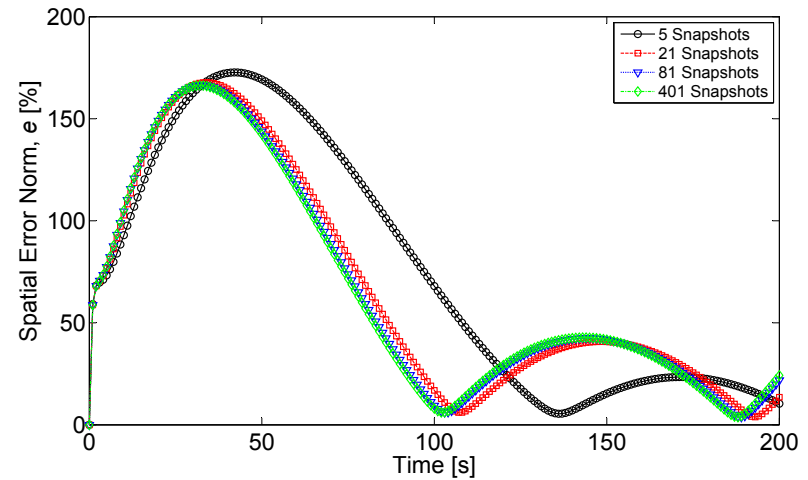

(b) Two basis vectors retained.

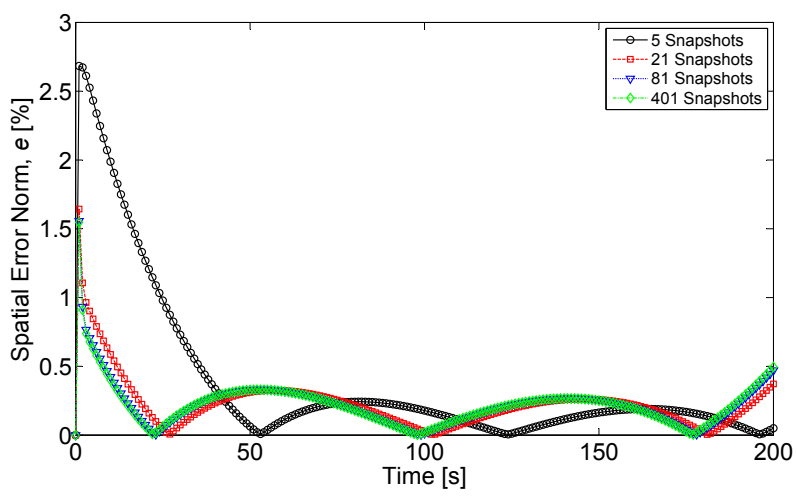

(c) Three basis vectors retained.

Figure 10. POD percentage error for varying number of snapshots and retained number of basis vectors.

From the results shown, one cannot conclude that taking more snapshots will result in a smaller error for any arbitrary time instant regardless of the number of basis vectors retained. Though in general it appears that taking more snapshots is advantageous, for certain time intervals the error is lower for the cases of fewer snapshots. To understand the underlying causes of this, the effect of number of snapshots on the POD error was investigated at the specific time instant of $200 \mathrm{~s}$. POD bases were generated for cases with varying numbers of snapshots ranging from 5 to 401. For each case, the error of the POD solution using 3 POD basis vectors at $200 \mathrm{~s}$ was calculated.

From Fig. 11, one can see that the increase in error with number of snapshots is in agreement with the results shown in Fig. 10 at $200 \mathrm{~s}$ in that the error increases asymptotically with number of snapshots. To further investigate the error of the POD basis as a function of number of snapshots, the relative energy of the excluded basis vectors given by Eq. (28) was calculated for varying number of snapshots as shown in Fig. 12. Again, three basis vectors were retained and the number of snapshots ranged from $5-401$.

The trend in Fig. 12 is similar to that in Fig. 11 and indicates that the energy of the first three POD vectors relative to that of the full set decreases asymptotically with increasing number of snapshots. It is believed that the reason for the increase in error with number of snapshots is due to spreading of the energy to higher modes with increasing number of snapshots. The number of POD basis vectors obtained is equal to the number of snapshots taken. Because taking more snapshots results in more POD basis vectors being created, it is possible that as more snapshots are taken, the proportion of energy contained in the first three basis vectors relative to the total energy of the set may decrease. The energy of the first three POD modes increases at a slower rate than the that of the higher modes with number of snapshots for this particular case. 


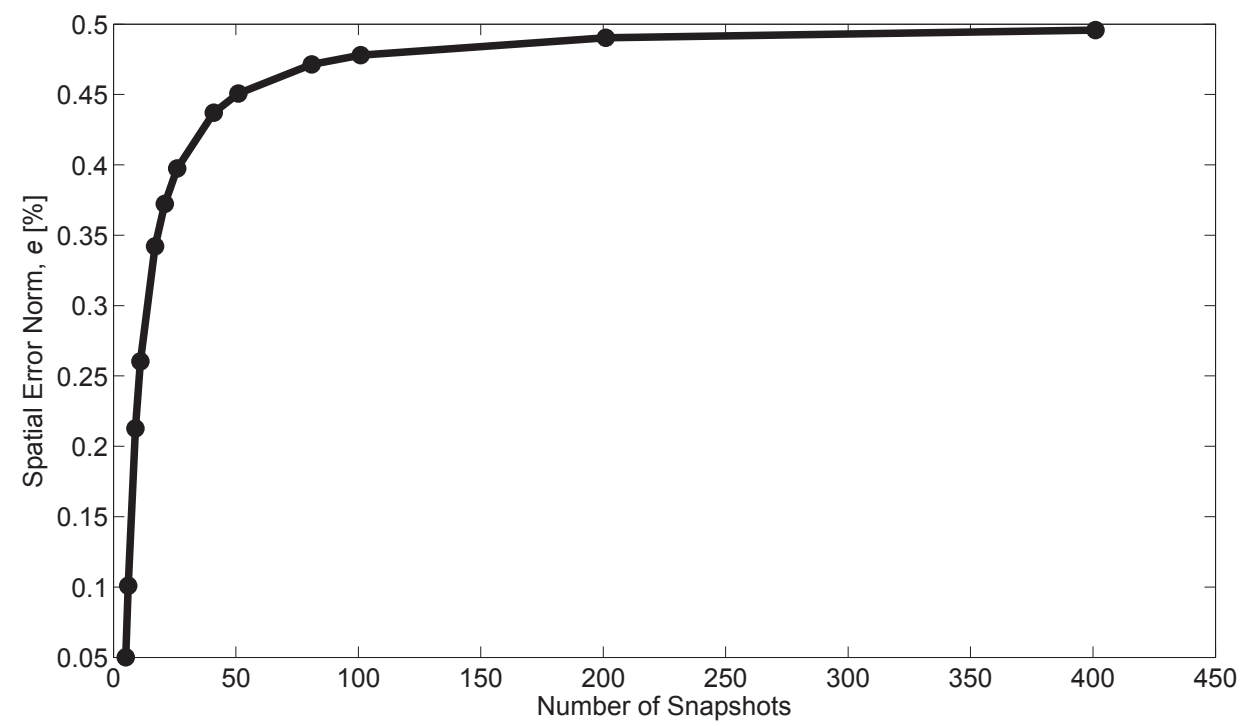

Figure 11. POD error for varying number of snapshots at $200 \mathrm{~s}$.

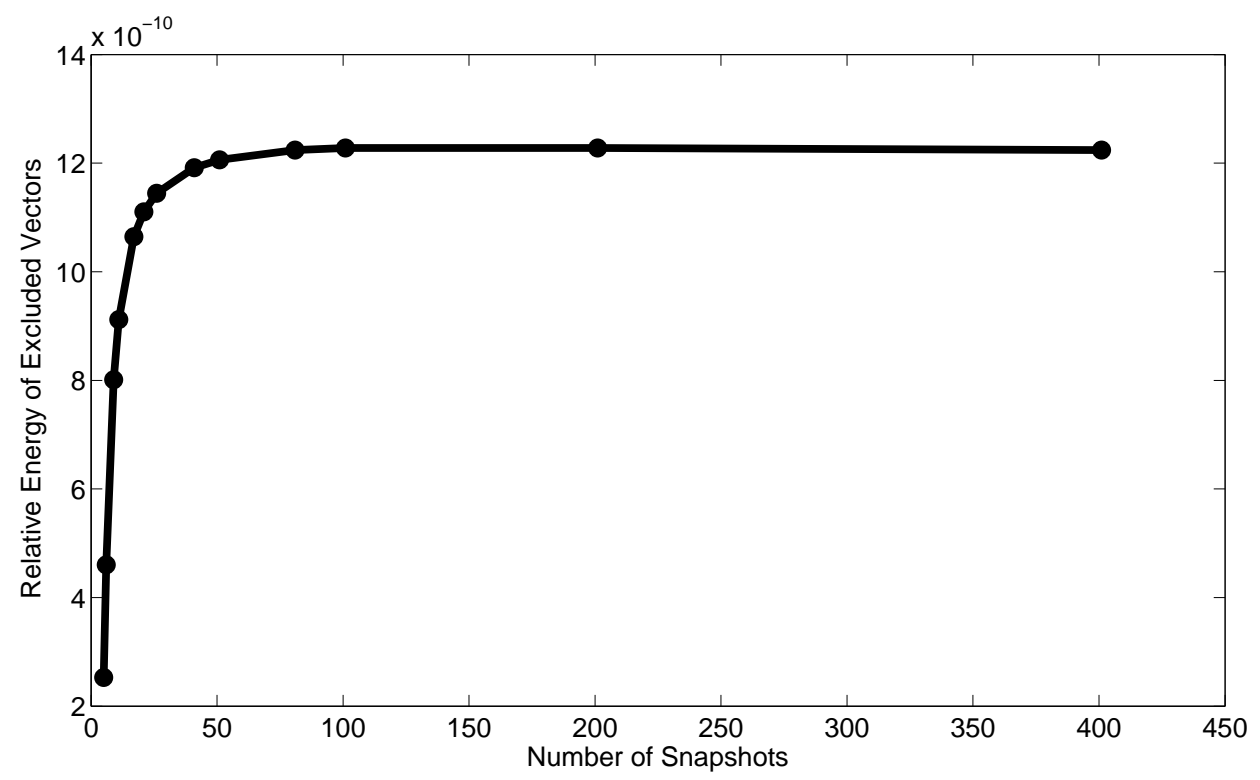

Figure 12. Relative energy of excluded basis vectors for varying number of snapshots at $200 \mathrm{~s}$. 
To understand the relevance of each of the first three POD modes to the solution, the modal coordinates of these modes were plotted as a function of number of snapshots again at $200 \mathrm{~s}$ and the results are given in Fig. 13. Again, in this case only the first three modes were used for the solution. Although the change is small, the modal coordinate of the first POD vector decreases with increasing number of snapshots. Those of the second and third POD vectors increase with increasing number of snapshots. These trends indicate that some of the energy of the first POD mode is being spread to the higher POD modes as the number of snapshots increases.

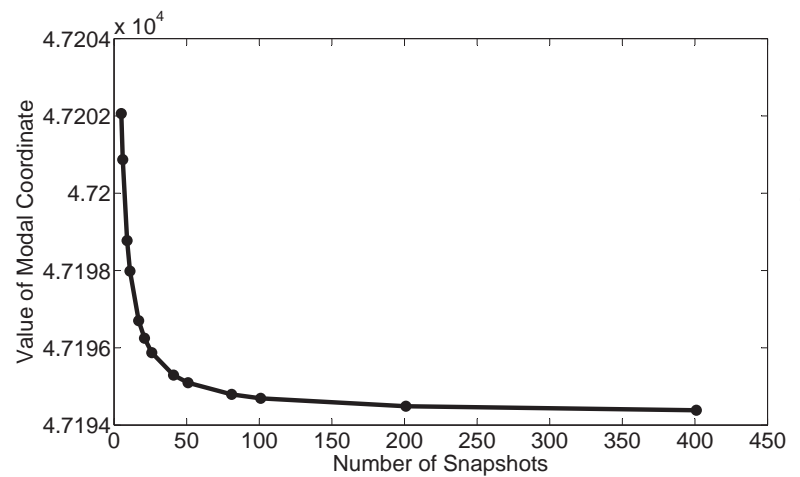

(a) Modal coordinate of first POD basis vector.

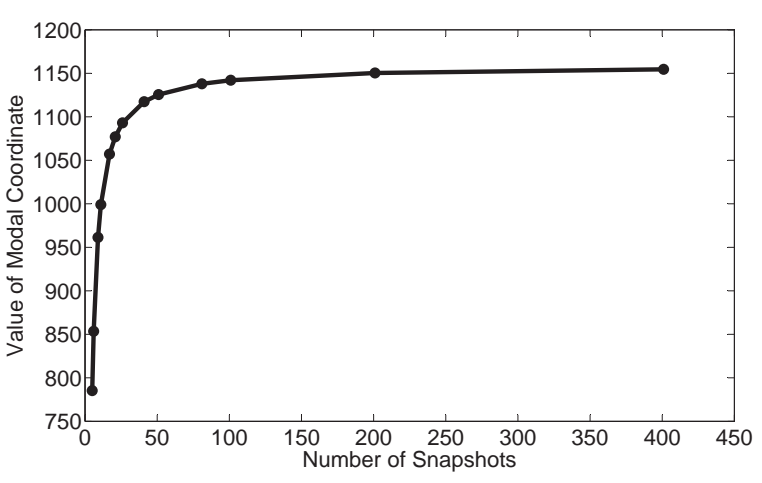

(b) Modal coordinate of second POD basis vector.

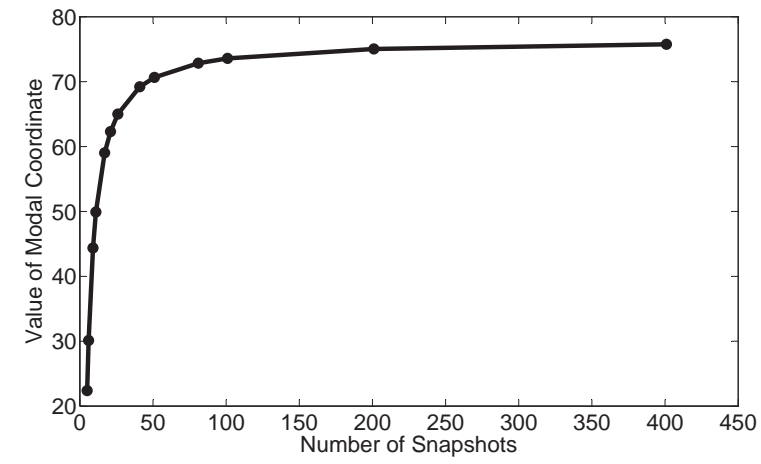

(c) Modal coordinate of third POD basis vector.

Figure 13. Modal coordinates of first three POD basis vectors for varying number of snapshots at $200 \mathrm{~s}$.

Finally, the utility of taking additional snapshots is measured by calculating the projections of the snapshots onto the subspaces spanned by the first snapshot, the first two snapshots, the first three snapshots, and the first four snapshots. Snapshots lying in the space already spanned by previous snapshots provide no additional information while snapshots orthogonal to the space already spanned by previous snapshots provide maximum information. The full QR factorization of the snapshot matrix is first computed such that $A=Q R$ where $Q$ is an orthogonal $s \times s$ matrix and $R$ is an upper triangular $s \times n$ matrix. The columns of $Q$ have the property that they span the same subspace as the corresponding columns of $A$ such that ${ }^{41}$

$$
\begin{aligned}
\left\langle q_{1}\right\rangle & =\left\langle a_{1}\right\rangle \\
\left\langle q_{1}, q_{2}\right\rangle & =\left\langle a_{1}, a_{2}\right\rangle \\
\left\langle q_{1}, q_{2}, q_{3}\right\rangle & =\left\langle a_{1}, a_{2}, a_{3}\right\rangle \\
& \vdots \\
\left\langle q_{1}, q_{2}, \ldots, q_{j}\right\rangle & =\left\langle a_{1}, a_{2}, \ldots, a_{j}\right\rangle,
\end{aligned}
$$

where $\langle\cdot\rangle$ indicates the subspace spanned by the vectors enclosed in the brackets. One can therefore use the columns of $Q$ to form orthonormal subspaces that span the subspaces of the corresponding columns of the snapshot matrix. Each snapshot is first normalized to unit magnitude, i.e.,

$$
\left\|\hat{a}_{i}\right\|_{2}=1, \quad i=1, \ldots, n,
$$


so that the magnitude of the projection of each snapshot onto the various subspaces will lie between zero and one. If we take $\hat{Q}$ to be the truncated version of $Q$ containing the first $j$ columns of $Q$, the projection onto the space spanned by $\hat{Q}$ is given by $\hat{Q} \hat{Q}^{T}$. If a snapshot $\hat{a}_{i}$ lies in the span of $\hat{Q}$, then applying the projection results in $\hat{a}_{i}$ itself, i.e., ${ }^{41}$

$$
\hat{Q} \hat{Q}^{T} \hat{a}_{i}=\hat{a}_{i}
$$

Define the residual, $\mathcal{R}$, as the normed difference between the projection of the $i$-th snapshot onto the subspace and the actual snapshot given by

$$
\mathcal{R}=\left\|\hat{Q} \hat{Q}^{T} \hat{a}_{i}-\hat{a}_{i}\right\|_{2}
$$

where a zero value of $\mathcal{R}$ indicates that the snapshot already lies in the subspace while a value of one indicates that the snapshot is orthogonal to the subspace. Results are given in Fig. 14 for projection of each of the snapshots onto the subspaces spanned by the first, first two, first three, and first four snapshots.

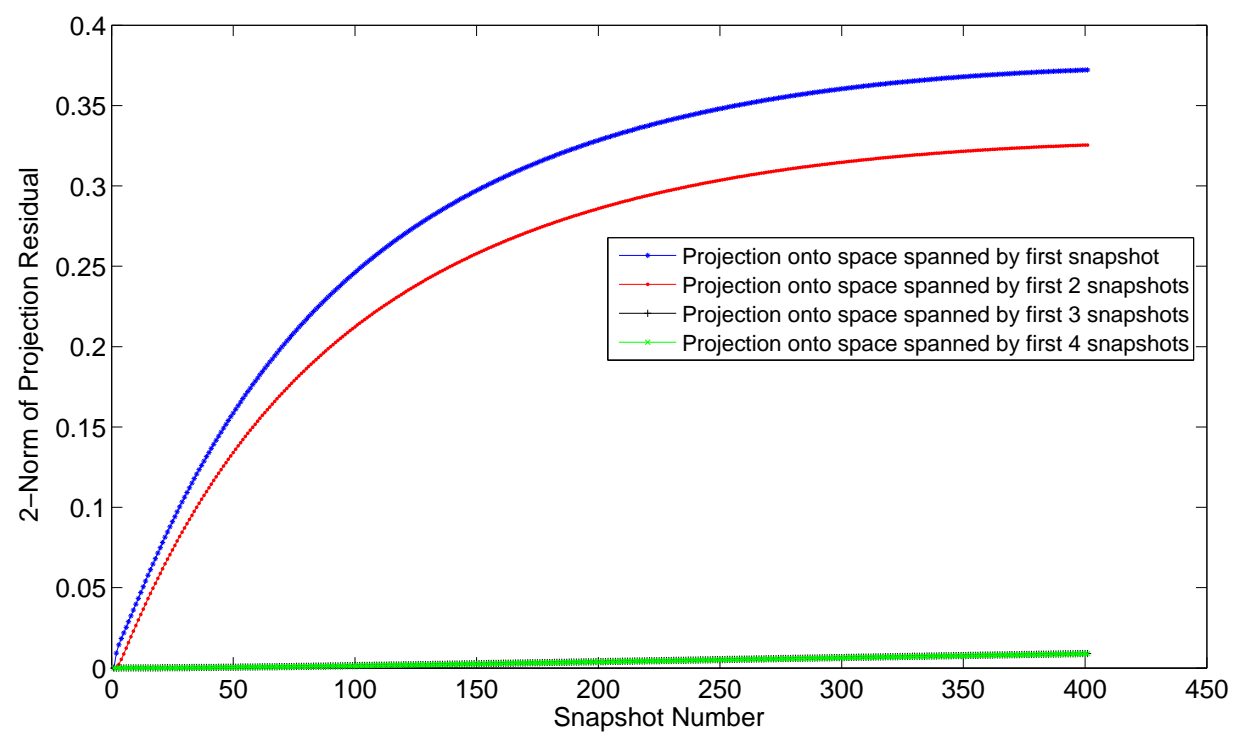

Figure 14. Residual of projection of snapshots onto various subspaces.

The results indicate that the space spanned by just the first three snapshots comes close to containing all of the snapshots. Projection of the snapshots onto the subspace spanned by the first four snapshots shows almost no noticeable difference from that onto the subspace spanned by the first three snapshots, indicating there is very little new information introduced by including the fourth snapshot. These results indicate that the first three snapshots capture most of the dynamics necessary for creation of the POD basis. This is not unexpected as the constant, uniform heat flux should lead to system dynamics which are easily captured as opposed to a case with more complex boundary conditions.

\section{IV.B. Pre-Specified Time-Dependent Thermal Load Vector}

The next case considered is that of a time-dependent thermal load vector whose functional form is specified a priori. One of the fundamental approximations to be examined in this case study is the use of a fixed POD basis for cases with time-varying natural boundary conditions. The advantage of using the same basis throughout the transient is that the need to perform the time-consuming computation of regenerating the basis during the course of a simulation is avoided. Additionally, the actual thermal loads on the HSV will not be known ahead of time, and the basis must therefore be robust to changes in the boundary conditions. The POD basis vectors are treated as Ritz vectors, similar to their use in structural dynamics. The basis vectors must only satisfy the Dirichlet boundary conditions ${ }^{66}$ (fixed-temperature boundary conditions in this case), and since the boundary conditions will only be of the Neumann type, the POD basis will satisfy the necessary conditions of Ritz modes throughout the transient. This case will be also used to compare the accuracy of the decoupled analytical POD solution with that of the numerically integrated POD solution with the goal of assessing the effect of the size of the time step on the numerical solution. This is important 
as the size of the time step used for the numerical solution of the thermal problem will dictate how frequently the thermal load vector must be updated. The form of the thermal load vector used for this case is given by

$$
F(x, y, z, t)=H(t) \tilde{g}(x, y, z),
$$

where $H(t)$ is a scalar time-dependent coefficient and $\tilde{g}(x, y, z)$ is a spatially varying vector. For this study, $H(t)$ is taken to be $\sin (\omega t)$ with $\omega=0.01 \mathrm{~Hz}$ and $\tilde{g}(x, y, z)$ is obtained from the uniform $10 \mathrm{~W} / \mathrm{cm}^{2}$ case above. The $i$-th decoupled equation will then be of the form

$$
\dot{\tilde{c}}_{i}(t)+\tilde{k}_{i} \tilde{c}_{i}(t)=\sin (\omega t) \tilde{g}_{i}
$$

and the solution subject to the initial condition $\tilde{c}(t=0)=0$ is given by

$$
\tilde{c}_{i}(t)=\frac{\tilde{g}_{i} \mathrm{e}^{-\tilde{k}_{i} t} \omega-\tilde{g}_{i} \omega \cos (\omega t)+\tilde{g}_{i} \tilde{k}_{i} \sin (\omega t)}{\tilde{k}_{i}^{2}+\omega^{2}} .
$$

The POD basis was first created by taking 501 snapshots in evenly spaced intervals between 0 and 500 seconds with one snapshot per second. Note that the snapshots were taken by decoupling the full system using the full eigendecomposition of the system and solving each equation analytically to eliminate any error that would be incurred due to numerical time-stepping. The temperature response from 0 to 2,000 $\mathrm{s}$ for a node at the outer surface of the heat shield, outer surface of the insulation layer, and on the bottom skin is given in Fig. 15.

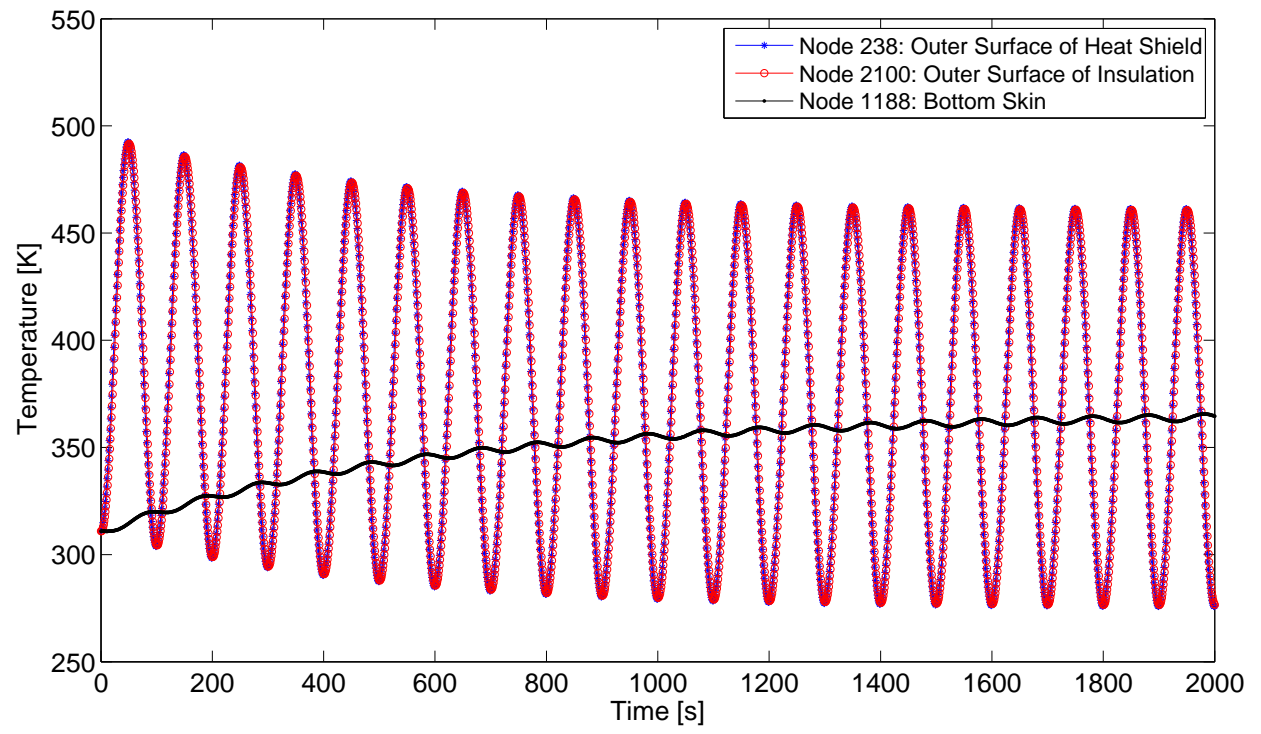

Figure 15. Temperature response for three selected nodes for the case of time-varying heat flux.

Once the snapshots were taken, the correlation matrix and its eigendecomposition were found. The magnitudes of the first nine eigenvalues of the correlation matrix are given in Fig. 16. Based on the eigenvalues of the correlation matrix, only the first four POD modes were retained in the solution. Note that the relative energy of the excluded POD modes as calculated using Eq. (28) is $1.58 \times 10^{-9}$. The first step was to compare the full-order solution with the solution obtained by introducing the POD modes, decoupling the equations, and solving them analytically, hereafter referred to as the POD-analytical solution. Using $e$ from Eq. (52), the error of the POD-analytical solution is given in Fig. 17 for the time interval in which the snapshots were taken (between 0 and $500 \mathrm{~s}$ ).

Though the POD-analytical solution shows good agreement with the full-order solution within the time range in which the snapshots were taken, it is likely that the reduced-order solution will need to extrapolate temperature distributions for time instants that are outside of the time interval in which the snapshots are taken for the actual HSV simulations. Thus, the POD-analytical solution was compared with the fullorder solution from 0 to 2,000 s to investigate the accuracy of the reduced-order solution outside of the time interval in which the snapshots were taken. Results for cases with three, four, five, and six POD basis vectors 


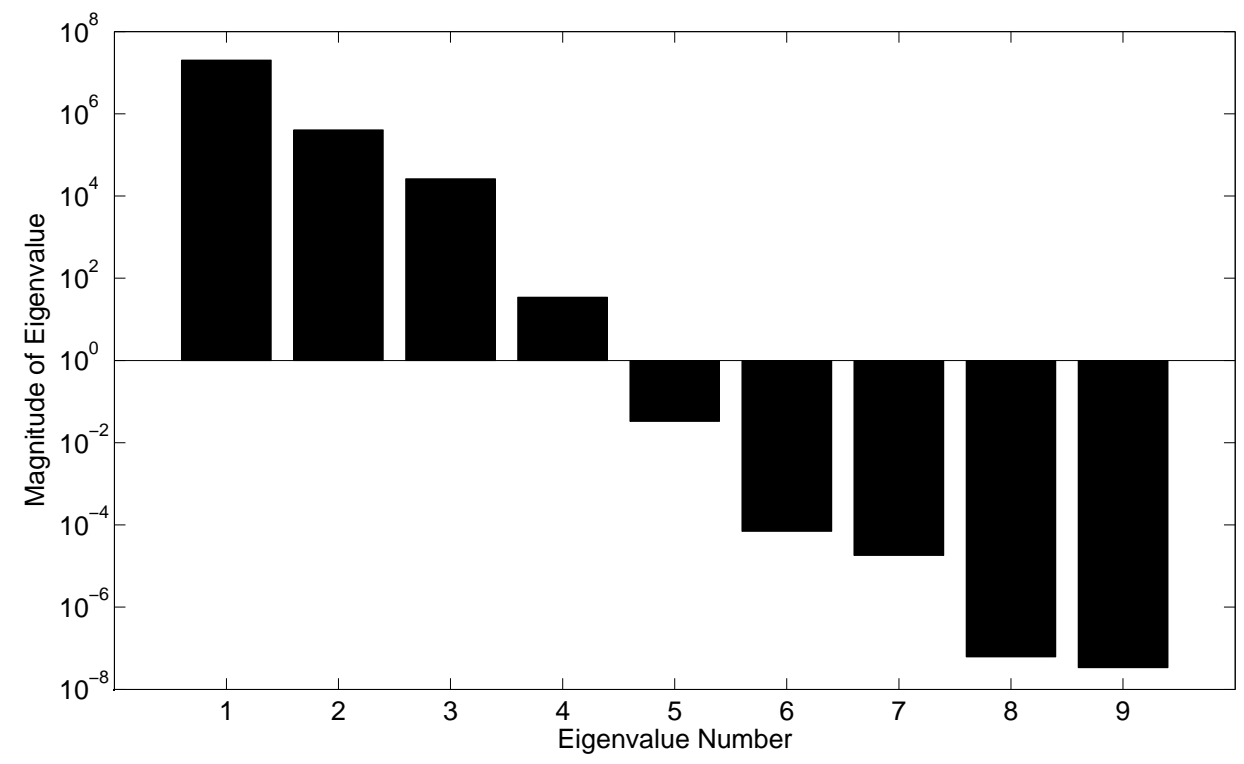

Figure 16. First nine eigenvalues of correlation matrix for the case of time-varying heat flux.

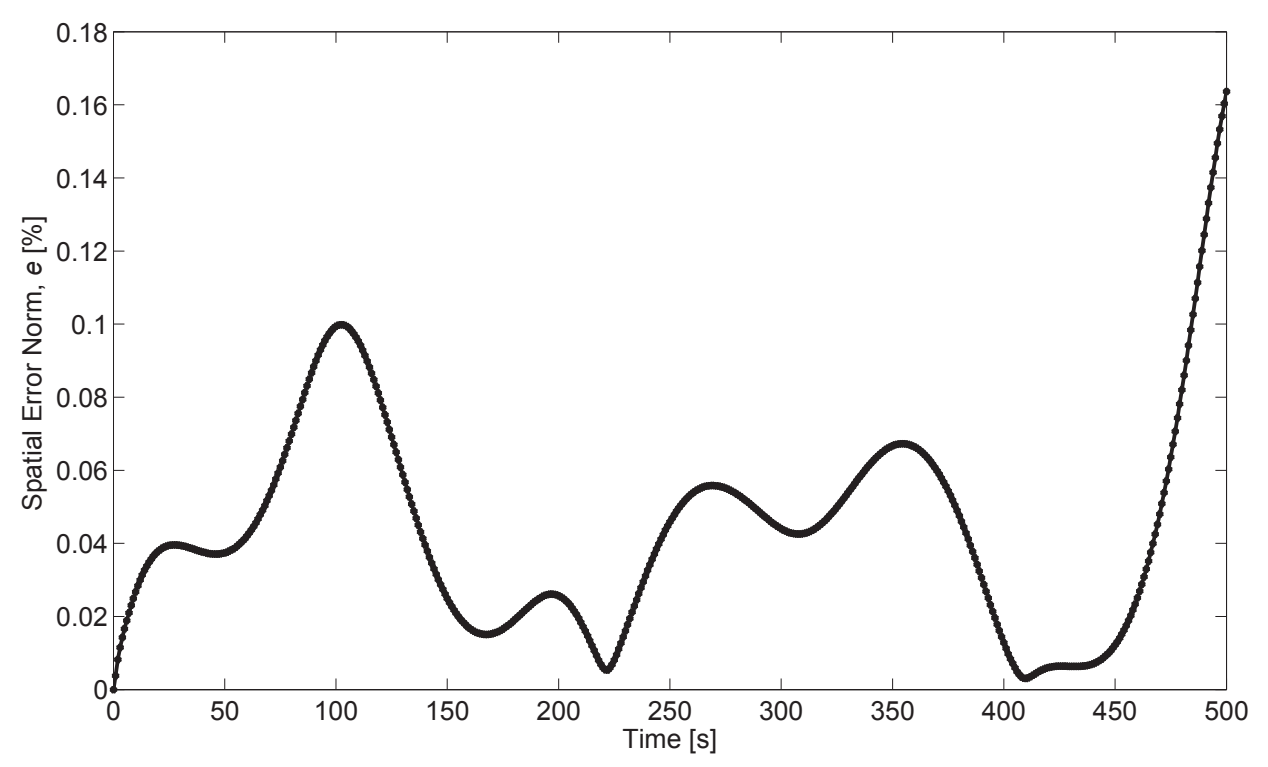

Figure 17. POD-analytical solution error. 
retained in the POD-analytical solution are given in Fig. 18. As seen in the figure, the overall accuracy of the reduced-order solution outside of the time range in which the snapshots were taken is lower than that within the time range in which the snapshots were taken. Furthermore, though retaining more than four POD basis vectors results in little improvement in solution accuracy between 0 and $500 \mathrm{~s}$, the use of additional basis vectors has a more prominent effect on the error for times beyond $500 \mathrm{~s}$.

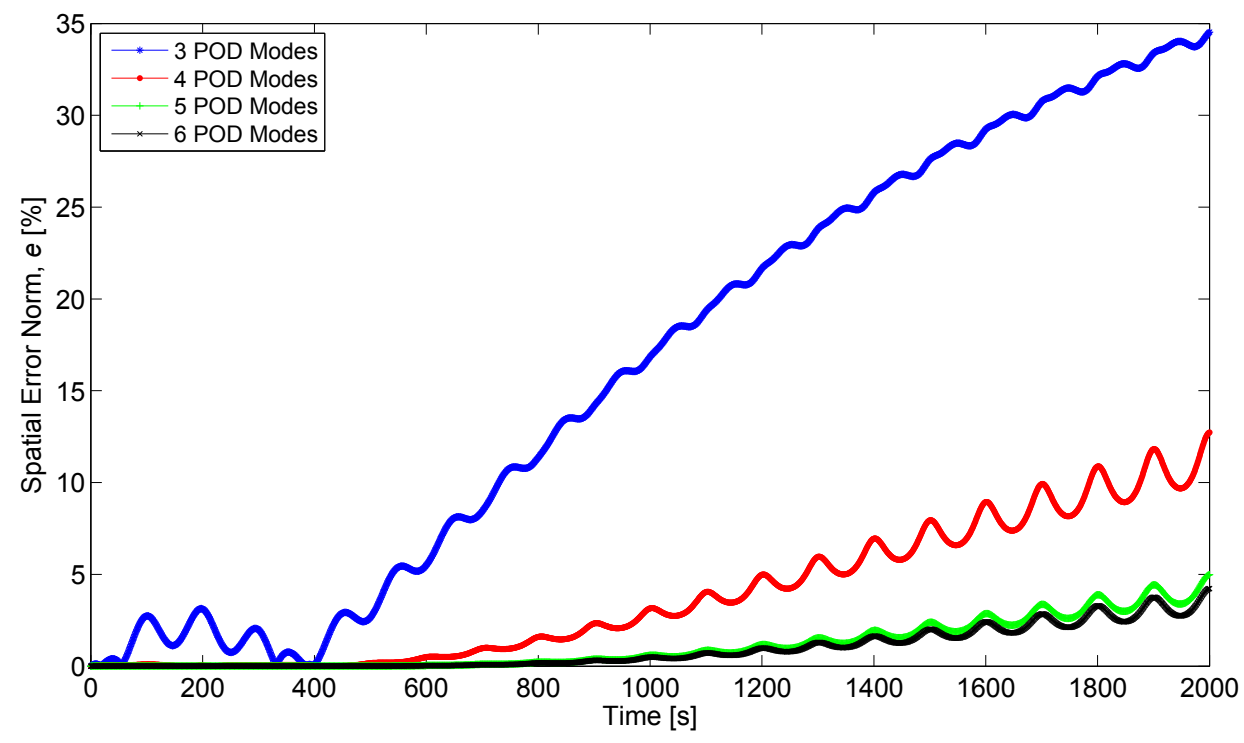

Figure 18. Error of POD-analytical solution from $0-2,000 \mathrm{~s}$ for varying number of basis vectors.

In actual aerothermoelastic simulations of HSVs, the time-dependence of the thermal boundary conditions will not be known ahead of time as the heat flux will depend on flow parameters which are influenced by the structural dynamic response. The simulations used to take the snapshots and create the POD basis may not therefore represent the thermal boundary conditions that the structure will see in the actual simulations. In order to most closely capture the space in which the solution is likely to lie, it is necessary to design the simulations to excite all of the system dynamics which are expected throughout the mission. To examine the effect of extracting POD modes from simulations with boundary conditions that are different from those present in the actual simulation, the POD basis from the previous constant heat flux case was used to obtain solutions for the current case of a pre-specified time-varying heat flux. The error between the full-order solution and POD-analytical was calculated between 0 - 2,000 s using varying number of basis vectors and results are given in Fig. 19. Note that the errors for the cases of four and five basis vectors retained are virtually identical. Comparing Fig. 19 with Fig. 18, it is observed that there is not a dramatic loss of error when the modes are taken from snapshots of simulations with boundary conditions that are different from those in the actual solution. Though more modes may need to be retained if the time-dependence of the boundary conditions is not known ahead of time, this result strengthens the case that it may be possible to use POD modes that are obtained a priori for the full aerothermoelastic HSVs simulations.

The accuracy of the coupled, numerically integrated POD solution, hereafter referred to as PODnumerical, is now investigated. The numerical solution is carried out using the Crank-Nicolson scheme as described previously using the first four POD basis vectors. Recall that the temperatures at the next time step $T_{n+1}$ depend on the thermal load vector at that next time step, $g_{n+1}$, as shown in Eq. (44). As we have specified the time-dependence of the thermal load vector ahead of time, for the first case we will use this information by calculating the actual value of $g_{n+1}$ and using it in the calculation of the temperatures, $T_{n+1}$. The transient temperature history for this case for node 238 which is approximately at the mid-chord, mid-span location on the bottom outer surface of the heat shield of the control surface is given in Fig. 20 for the full-order solution and POD-numerical solution with $\Delta t=10 \mathrm{~s}$ and $\Delta t=25 \mathrm{~s}$. Again, note that the full order solution is obtained analytically and thus does not contain numerical error. The error of the POD-numerical solution with respect to the full order solution was then calculated over time using the spatial error norm, e, for various time step sizes. The results are given in Fig. 21. 


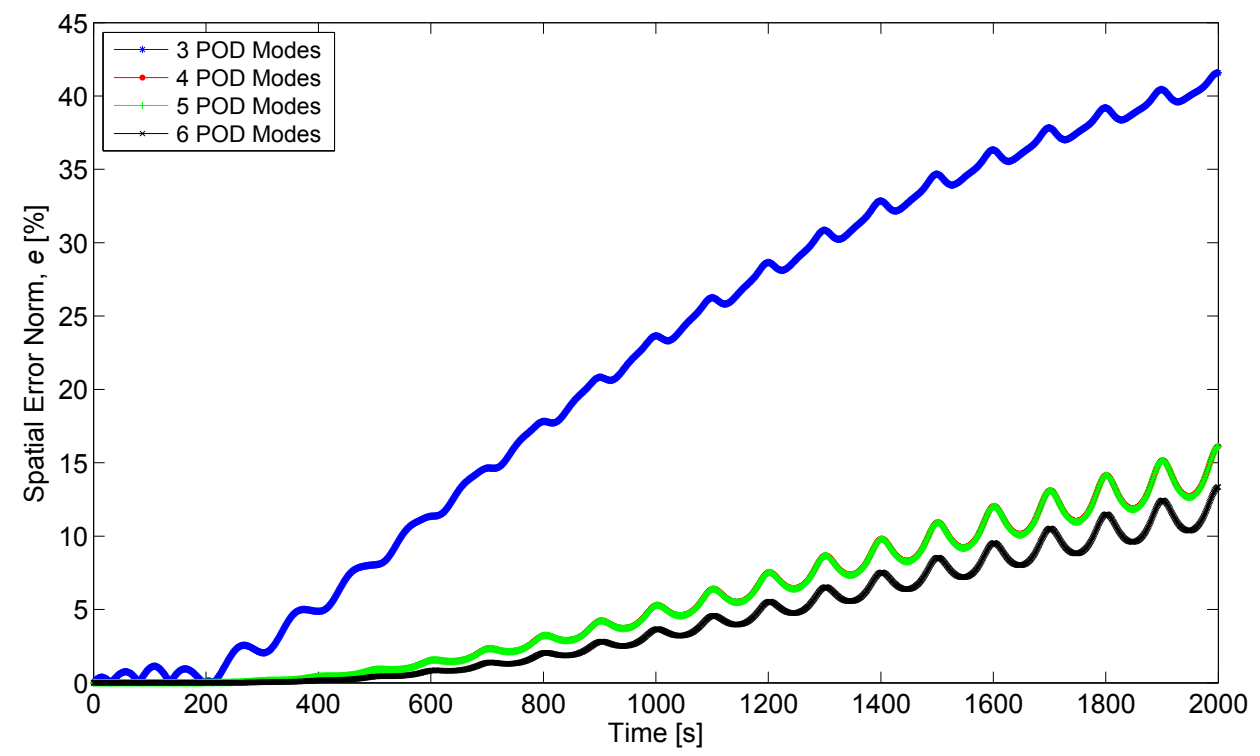

Figure 19. Error of POD-analytical solution from 0 to $2,000 \mathrm{~s}$ using modes from constant heat flux case.

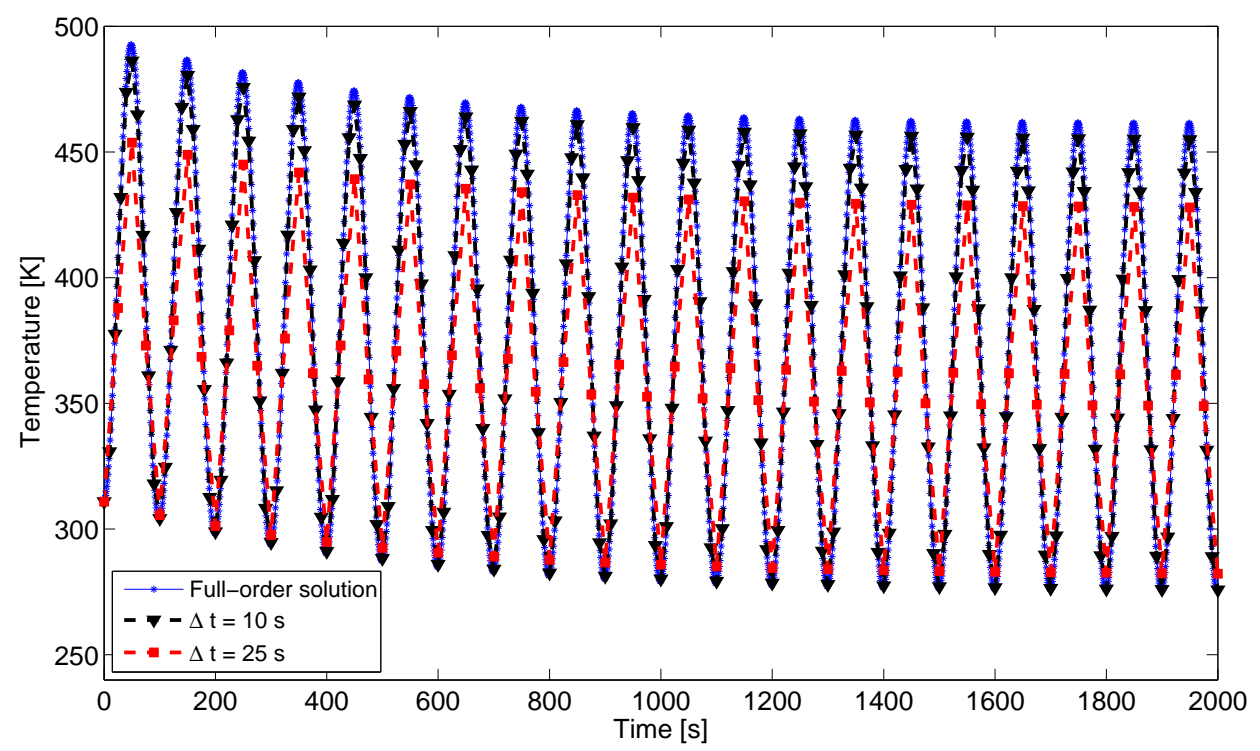

Figure 20. Temperatures at Node 238 for full order and POD-numerical solution with varying time step sizes. 


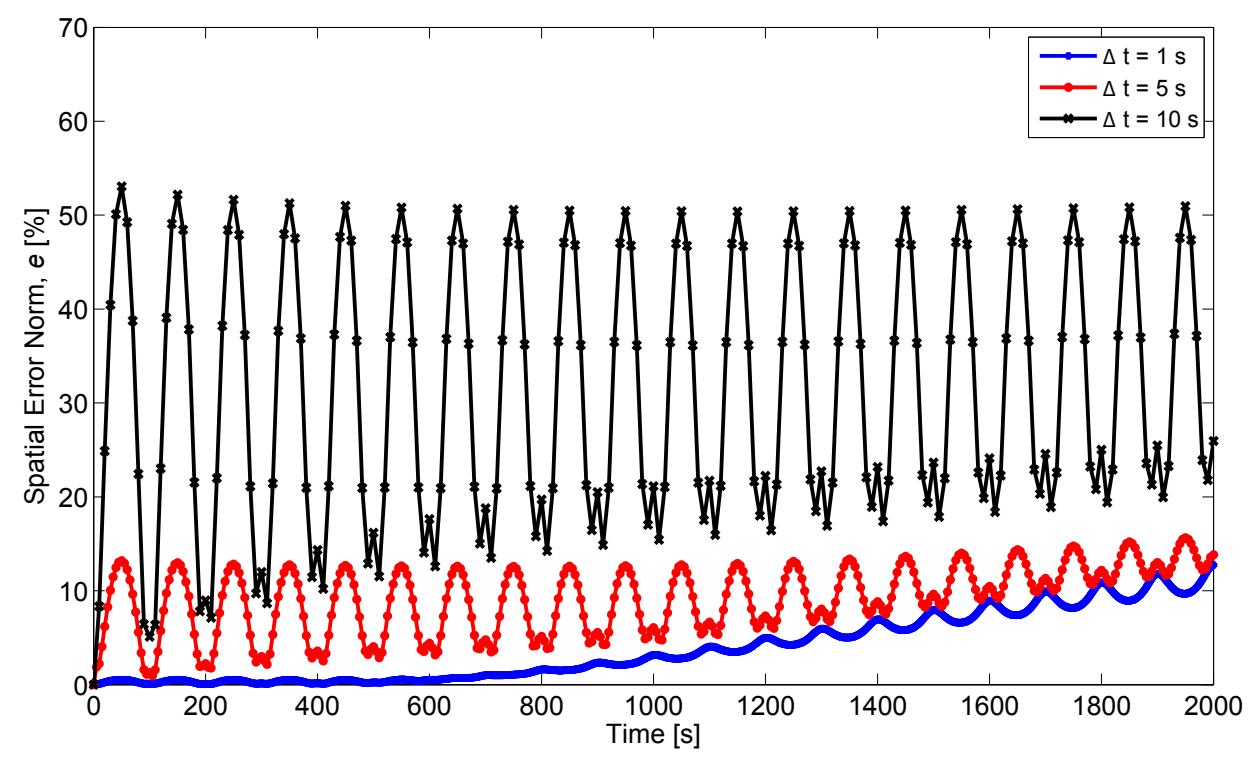

Figure 21. Error of POD-numerical solution using known time-dependence of thermal load vector.

While using the known time-dependence of the thermal load vector at the time instant at which the solution is desired results in reasonable accuracy, this is not possible within actual HSV aerothermoelastic simulations as the thermal load vector will not be known ahead of time. This is due to the fact that it depends on the instantaneous flow parameters, which change as the structure deforms. A nonlinear solution is undesirable as this would counteract the computational savings of using reduced-order models. As the cost of time-marching the transient thermal solution has been made relatively cheap through the use of POD, reducing the size of the time step and performing a linear solution at each time step is expected to achieve the desired computational efficiency while still maintaining reasonable accuracy. As such, the error of the POD-numerical solution was again calculated, except the problem was treated as if the thermal load vector is not known ahead of time. Instead, it was treated as piecewise constant such that $g_{n+1}=g_{n}$ between each set of time steps. Once the temperature is calculated at the current time instant, the load vector is updated and again assumed constant until the next update. The error of the POD-numerical solution with this approximation is given in Fig. 22 for various size time steps.

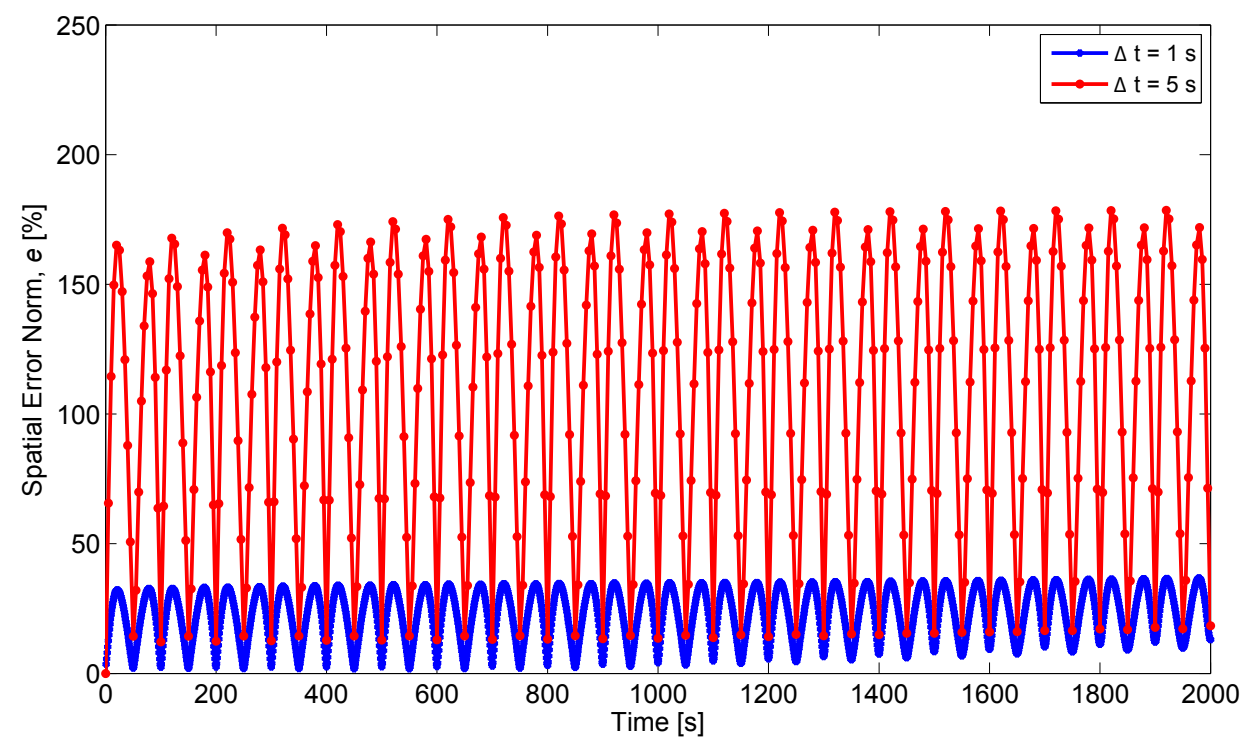

Figure 22. Error of POD-numerical solution with piecewise constant approximation to thermal load vector. 
Comparing Fig. 22 with Fig. 21, we see that the error introduced through the piecewise constant approximation of the thermal load vector can significantly increase the error of the POD-numerical solution. Thus, careful attention must be paid to the time step selection or alternative approximations should be made in the actual HSV simulations. One potential alternative to the piecewise-constant approximation would be to create a functional representation of the thermal loads in between updates based on the values at previous time instants.

\section{IV.C. Use of POD Within Aerothermoelastic Simulation Framework}

A time-marching procedure with updates to the thermal and structural boundary conditions at specified intervals is proposed for solution of the coupled aerothermoelastic problem. This approach is based on an extension of the quasi-steady formulation utilized in a previous work ${ }^{67}$ to include unsteady aerodynamic effects and inertial effects due to structural dynamics. An outline of the time-stepping schedule is given in Fig. 23.

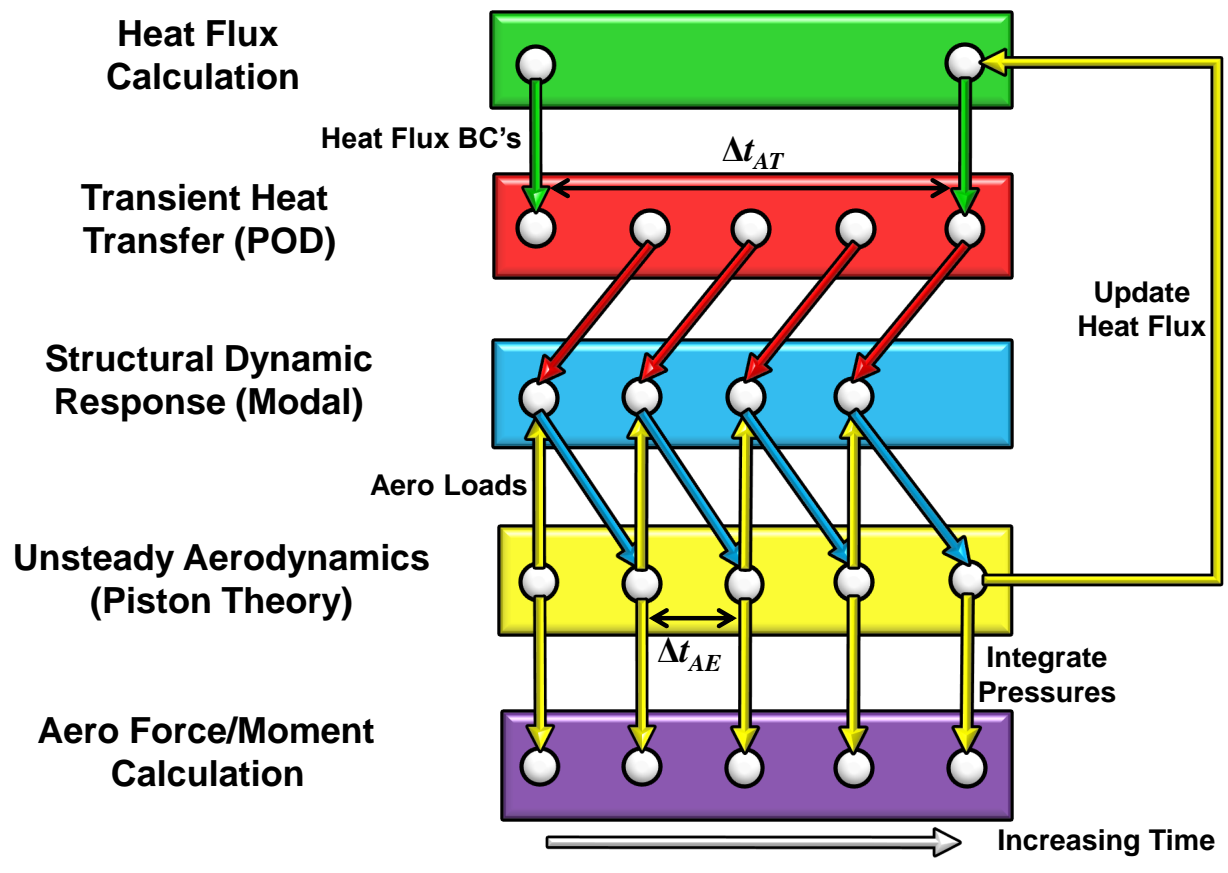

Figure 23. Overview of aerothermoelastic time-stepping schedule.

A fundamental assumption to be investigated in the study is that the size of the aeroelastic time step, $\Delta t_{A E}$, will be smaller than the size of the aerothermal time step, $\Delta t_{A T}$. The procedure begins by calculating the aerodynamic flow properties over the undeformed structure at initial time. The heat flux is then calculated which gives the skin friction coefficient to be used in the viscous drag calculation. The aerodynamic pressures and viscous drag are then integrated to determine the aerodynamic forces and moments at initial time. A pre-determined number of thermal time steps (four in this example), each of size $\Delta t_{A E}$, are then taken and the thermal loads from the first thermal step are applied to the undeformed structure. Additionally, the aerodynamic loads based on the already calculated flow properties are applied to the structure. The structural deformations are then calculated. The displacements are fed back into the aerodynamic solver and the flow properties are calculated at time $t+\Delta t_{A E}$ over the updated deformed configuration. At each aeroelastic time step, the updated aerodynamic pressure loads and thermal loads are applied to the structure. Additionally, each time the flow properties are re-calculated, the aerodynamic pressures are integrated to allow for characterization of the transient aerodynamic forces and moments on the vehicle. The aeroelastic iterations continue to be carried for a pre-determined number of time steps. Once the time instant $t+4 \Delta t_{A E}$ has been reached, the aerodynamic flow properties at the current time instant are used to update the aerodynamic heat flux. With the updated thermal boundary conditions known, the transient thermal solution is marched forward another four time steps and the process is repeated. The procedure of time-marching the various solutions involved is a major aspect of this study. Specific parameters to be investigated involve the 
number of aeroelastic time steps before updating the thermal boundary conditions and the size of the aeroelastic and aerothermal time steps. Though results are not ready for publication at this time, the following sections describe the formulations for solution of the various aspects of the aerothermoelastic problem.

\section{IV.C.1. Reduced Order Modal Basis Solution for Structural Dynamic Response}

The problem of solving for the structural dynamic response of hypersonic vehicle structures within a control design and evaluation framework is complicated by various factors. Due to the large number of degrees of freedom involved in a traditional finite element solution, steps must be taken to reduce the order of the structural dynamics system of equations. A common approach is to employ a modal transformation in which the structural displacements are written as a linear combination of small number of basis vectors which are the free vibration mode shapes of the structure. However, this approach cannot be applied directly for hypersonic vehicle problems as the mode shapes change over time due to geometric stiffness and material degradation effects. The approach taken in this work is to first perform an off-line calculation and select a reduced number of Ritz modes based on the free vibration modes of the structure at a reference temperature distribution. These Ritz modes will then be used as the modal basis for solution of the structural response throughout the simulation. This procedure is applicable as the Ritz modes need only to satisfy the geometric boundary conditions, ${ }^{66}$ which will always be the case regardless of the stiffness distribution. The reference modes, $\Phi_{r e f}$, will not be updated during the simulation, thus avoiding the solution of an eigenvalue problem of the full system at each time step. Consider the system of structural dynamics equations of motion given by

$$
M_{s} \ddot{x}+K_{s}^{*}(T) x=F_{s}(t),
$$

where $M_{s}$ is the mass matrix, $F_{s}$ is the load vector, $x$ are the physical degrees of freedom. The modified stiffness matrix, $K_{s}^{*}$, is given by

$$
K_{s}^{*}(T) \equiv K_{s}(T)+K_{G}(T),
$$

where $K_{s}(T)$ is the conventional stiffness matrix that varies due to the temperature-dependence of the material properties and $K_{G}(T)$ is the geometric stiffness matrix resulting from thermal stresses. Though the reference modes will not be updated throughout the simulation, the modified stiffness matrix will be updated at pre-determined time intervals to account for the dependence on temperature. Updating of the conventional stiffness matrix is performed using the functional dependence of the Young's Moduli of the various materials. The geometric stiffness matrix is updated by solving a static finite element problem based on the thermal loads from temperatures at the current time step and the corresponding material coefficients of thermal expansion. The physical degrees of freedom are expressed as a linear combination of the reference free vibration modes such that

$$
x=\Phi_{r e f} \zeta,
$$

where $\zeta$ represents the modal coordinates of the reference modes. Once the modified stiffness matrix is known at the current time instant, the system is reduced by substituting Eq. (62) into Eq. (60) and pre-multiplying the system by $\Phi_{r e f}^{T}$, i.e.,

$$
\Phi_{r e f}^{T} M_{s} \Phi_{r e f} \ddot{\zeta}+\Phi_{r e f}^{T} K_{s}^{*}(T) \Phi_{r e f} \zeta=\Phi_{r e f}^{T} F_{s}(t) .
$$

As the mass of the structure will be taken to be constant in this work, the reference modes will be orthogonal with respect to the mass matrix and the product $\Phi_{r e f}^{T} M_{s} \Phi_{r e f}$ will reduce to the identity matrix. Since the modified stiffness matrix will be continuously changing, we have no guarantee of orthogonality of the reference modes with respect to stiffness, and the equations will be coupled. As such, a numerical integration routine will be employed to calculate the vector of modal coordinates at each time instant. Note that since the number of reference modes to be used in the modal expansion will be much less than the number of physical degrees of freedom in the model, the computational cost of the numerical solution of the system will be relatively low.

\section{IV.C.2. Heat Flux Calculation}

In order to obtain the temperature distribution associated with the thermal load applied to the structure, the transient heat transfer problem must first be solved. Solution of the heat transfer problem requires boundary conditions at the outer surfaces of the model and initial temperature conditions. For the initial condition, the structure is assumed to be at a uniform temperature of $311 \mathrm{~K}\left(100^{\circ} \mathrm{F}\right)$. For the boundary conditions, 
this study considers a convective heat flux due to aerodynamic heating, and radiation to the atmosphere at the outer surface. As this analysis considers a cruise trajectory at $26 \mathrm{~km}(85,000 \mathrm{ft})$, the atmospheric temperature is taken to be constant at $222.5 \mathrm{~K}$.

In order to calculate the spatially varying heat flux resulting from aerodynamic heating, the local freestream pressure, temperature, and Mach number must first be found. To calculate the initial values of these properties, an aerodynamics solution (to be described in the subsequent section) is generated based on the undeformed configuration of the model. Once the values of these aerodynamic parameters are known at each node of the model, the values from corresponding nodes of each element are averaged and treated as constant over the element so that an element-uniform heat flux can be calculated. From Holman, ${ }^{68}$ the convective heat flux at the surface is given by

$$
\dot{q}_{a e r o}=h\left(T_{w}-T_{r}\right),
$$

where $h$ is the heat transfer coefficient, $T_{w}$ is the wall temperature, and $T_{r}$ is the recovery temperature or adiabatic wall temperature. Because the process that brings the fluid to rest in the boundary layer is irreversible, and because not all of the free-stream kinetic energy is converted to thermal energy in this process, ${ }^{68}$ a recovery factor is used, which is given by

$$
r_{f}=\frac{T_{r}-T_{e}}{T_{t}-T_{e}}
$$

where $T_{e}$ is the temperature at the outer edge of the boundary layer and $T_{t}$ is the total temperature or stagnation temperature. The total temperature is given by

$$
T_{t}=T_{e}\left[1+(\gamma-1) \frac{M_{e}^{2}}{2}\right],
$$

where $M_{e}$ is the Mach number at the edge of the boundary layer, $T_{e}$ is the temperature at the edge of the boundary layer, and $\gamma$ is the ratio of specific heats which is taken to have the constant value of 1.4 in this study. Note that the local pressure at the edge of the boundary layer will be calculated from piston theory. However, the local temperature and Mach number at the edge of the boundary layer are both required to find the total temperature. To find these properties, isentropic flow is assumed between the leading edge and the location of interest on the surface of the structure. For an isentropic process, the total pressure and total temperature remain constant and one can write

$$
\begin{aligned}
& p_{t}=p_{\infty}\left(1+\frac{\gamma-1}{2} M_{\infty}^{2}\right)^{\frac{\gamma}{\gamma-1}}=p_{e}\left(1+\frac{\gamma-1}{2} M_{e}^{2}\right)^{\frac{\gamma}{\gamma-1}} \\
& T_{t}=T_{\infty}\left(1+\frac{\gamma-1}{2} M_{\infty}^{2}\right)=T_{e}\left(1+\frac{\gamma-1}{2} M_{e}^{2}\right)
\end{aligned}
$$

where $p_{t}$ is the total pressure, $p_{\infty}, T_{\infty}$, and $M_{\infty}$ are the freestream flow properties, and $p_{e}$ and $M_{e}$ are the local flow properties at the edge of the boundary layer. Using these relations, we can then express ratios between the freestream pressure and temperature and the local pressure and temperature as

$$
\begin{aligned}
\frac{p_{e}}{p_{\infty}} & =\left(\frac{1+\frac{\gamma-1}{2} M_{\infty}^{2}}{1+\frac{\gamma-1}{2} M_{e}^{2}}\right)^{\frac{\gamma}{\gamma-1}} \\
\frac{T_{e}}{T_{\infty}} & =\frac{1+\frac{\gamma-1}{2} M_{\infty}^{2}}{1+\frac{\gamma-1}{2} M_{e}^{2}} .
\end{aligned}
$$

This study assumes turbulent flow, thus the recovery factor is given by

$$
r_{f}=\operatorname{Pr}^{1 / 3},
$$

where the Prandtl number, $P r$, is approximated by assuming a constant value of $0.7 .^{68}$ Due to the large property variations across the boundary layer in the hypersonic flow, the constant-property heat transfer 
relations are used with the properties evaluated at a reference temperature, $T^{*}$, as proposed by Eckert. ${ }^{69}$ The reference temperature is given by

$$
T^{*}=T_{e}+0.50\left(T_{w}-T_{e}\right)+0.22\left(T_{r}-T_{e}\right) .
$$

Once the reference temperature is known, the reference density, $\rho^{*}$, and reference viscosity, $\mu^{*}$, are calculated using the ideal gas law and Sutherland's law, respectively. With these reference quantities known, the Reynolds number is found using

$$
R e_{x}^{*}=\frac{\rho^{*} V_{e} x_{d}}{\mu^{*}},
$$

where $x_{d}$ is the distance from the leading edge and $V_{e}$ is the velocity of the flow at the outer edge of the boundary layer. With the local Reynolds number known, the skin friction coefficient, $c_{f}^{*}$, is calculated using the Schultz-Grunow relation which accurately represents measurements with Reynolds numbers up to $10^{9}$ and is given by ${ }^{69}$

$$
c_{f}^{*}=\frac{0.370}{\left(\log _{10} R e_{x}^{*}\right)^{2.584}} .
$$

Once the skin friction is known, the reference Stanton number can be found using the Colburn-Reynolds analogy,

$$
S t^{*}=\frac{c_{f}^{*}}{2} \operatorname{Pr}^{-2 / 3},
$$

and the heat transfer coefficient is found using the definition of Stanton number,

$$
h=S t^{*} c_{p} \rho^{*} V_{e}
$$

Note that the flow is assumed to be calorically perfect for the purposes of this study and thus a constant value of specific heat, $c_{p}$, is used. Using the heat flux and radiation boundary conditions along with the initial temperatures, the temperature distribution is propagated forward in time using a transient thermal solution.

\section{IV.C.3. Unsteady Aerodynamic Modeling}

To capture the unsteady aerodynamic effects due to oscillation of the airfoil, piston theory will be used for calculation of the aerodynamic pressures acting on the outer surface of the structure. This theory provides a closed form expression which relates the local pressure resulting from a body's motion to the normal component of the fluid velocity at the location of interest. Early development of piston theory was performed by Lighthill ${ }^{70}$ who discussed its application to oscillating airfoils. Ashley and Zartarian ${ }^{71}$ further discussed the theory and applied it to a variety of aeroelastic problems. The fundamental underpinning of this aerodynamic theory is that in a two-dimensional inviscid flow, a perpendicular column of fluid stays intact as it passes over the surface of a structure. ${ }^{72}$ Thus, the unsteady pressure is a calculated at a specific location as if it were the face of a piston moving into a one-dimensional channel. The pressure on the face of a piston moving in a channel of perfect gas with velocity $V_{n}$ is given by ${ }^{71}$

$$
\frac{p}{p_{\infty}}=\left(1+\frac{\gamma-1}{2} \frac{V_{n}}{a_{\infty}}\right)^{\frac{2 \gamma}{\gamma-1}}
$$

where $p$ is the surface pressure, $p_{\infty}$ is the freestream pressure, $V_{n}$ is the velocity of the surface normal to the flow, and $a_{\infty}$ is the freestream speed of sound. Due to the hypersonic flow regime considered in this work, third order piston theory will be utilized. ${ }^{73}$ The pressure coefficient based on third order expansion of Eq. (75) is given by ${ }^{74}$

$$
C_{p}=\frac{2}{M_{\infty}^{2}}\left[\frac{V_{n}}{a_{\infty}}+\frac{(\gamma+1)}{4}\left(\frac{V_{n}}{a_{\infty}}\right)^{2}+\frac{(\gamma+1)}{12}\left(\frac{V_{n}}{a_{\infty}}\right)^{3}\right] .
$$

The pressure coefficient for compressible flow is given by ${ }^{75}$

$$
C_{p}=\frac{2}{\gamma M_{\infty}^{2}}\left(\frac{p}{p_{\infty}}-1\right)
$$


Using Eq. (77) in Eq. (76), we obtain ${ }^{76}$

$$
p-p_{\infty}=\gamma p_{\infty}\left[\frac{V_{n}}{a_{\infty}}+\frac{(\gamma+1)}{4}\left(\frac{V_{n}}{a_{\infty}}\right)^{2}+\frac{(\gamma+1)}{12}\left(\frac{V_{n}}{a_{\infty}}\right)^{3}\right] .
$$

Note that $V_{n}$ is given in terms of the freestream velocity, $U_{\infty}$, and the position of the outer surface of the structure, $Z(x, y, t)$, by

$$
V_{n}=\frac{\partial Z(x, y, t)}{\partial t}+U_{\infty}\left[\frac{\partial Z(x, y, t)}{\partial x}+\alpha\right]
$$

where $x$ is the freestream direction, $Z(x, y, t)$ is the position of the surface of the structure, and $\alpha$ is the angle-of-attack. Note that $Z(x, y, t)$ is a function of the elastic deformation of the structure and the geometry of the outer mold line and is given by

$$
Z(x, y, t)=w_{d}(x, y, z, t)+Z_{s t r}(x, y),
$$

where $w_{d}$ is the displacement in the $z$ direction normal to the flow direction and $Z_{s t r}(x, y)$ is a function describing the geometry of the outer mold line of the structure. Calculation of the spatial and temporal partial derivatives in Eq. (79) are performed using finite difference approximations at the nodal locations of the finite element grid.

\section{Concluding Remarks}

A reduced-order formulation for solution of the transient heat transfer problem based on POD has been given. This method has been chosen due to the optimality of the POD basis in representing the dominant modes of a system with the smallest possible number of basis vectors. The basis has been obtained in this work by taking snapshots of the solution over time from representative full-order simulations and detecting the correlation between snapshots. The solution of the reduced-order system of transient thermal equations resulting from projection onto the truncated basis was carried out using two different methods: one in which the equations were decoupled and solved analytically and another in which the equations were numerically integrated directly. The methodology was applied to a representative hypersonic vehicle elevator model as such a control surface is expected to have a significant contribution to the dynamics of the vehicle.

The first case analyzed was that of a time-independent, uniform thermal load vector. Results indicated good agreement between the full-order and reduced-order solutions. Investigation into the effect of number of snapshots on the solution indicated that the error incurred in the reduced-order solution does not always decrease with number of snapshots. This can be at least partly attributed to the increase in relative energy of the excluded basis vectors with increasing number of snapshots. Additionally, it was shown that for this case, the contribution of additional snapshots degrades rapidly after approximately three snapshots due to the fact that subsequent snapshots come close to lying in the subspace already captured by the first three snapshots.

Application of the methodology to a case with a pre-specified time-varying thermal load vector allowed for investigation of the error incurred by not updating the basis as the natural boundary conditions change. Results from the POD-analytical solution showed good accuracy in the time range considered by the snapshots, however the error was found to increase outside of the time range considered by the snapshots. Use of the basis from the constant heat flux case for solution in the time-varying case showed that although there is an increase an error, acceptable accuracy may be obtained even if the time-dependence of the boundary conditions is not known a priori. This provides support of the use of the same basis throughout hypersonic aerothermoelastic simulations. Solution of the reduced-order system using a numerical integration scheme showed that the accuracy is strongly dependent on the size of the time step chosen. Furthermore, results indicate that investigation into methods for approximating the time-dependence of the thermal loads in between updates may be warranted.

A reduced-order hypersonic aerothermoelastic simulation framework was described in which the POD formulation is used for the thermal aspect of the solution. The framework is a time-marching scheme in which the thermal and structural boundary conditions are updated at different rates. A reduced-order modal method is used for solution of the structural dynamics equations of motion. The aerodynamic heat flux boundary conditions are calculated using a first-principles Eckert reference temperature formulation. 
The unsteady aerodynamic loads are computed using a third-order piston theory expansion. The goal for the thermal component of this framework is to create a POD basis that is robust enough to be used throughout the simulation. This eliminates the need for solution of large-scale heat transfer solutions during the course of the simulation. The use of proper orthogonal decomposition for transient thermal solution will contribute to reducing the computational burden and number of states in hypersonic aerothermoelastic simulations.

\section{Acknowledgment}

This work was supported by the Michigan-AFRL Collaborative Center in Control Science (MACCCS) under grant number FA 8650-07-2-3744 (Air Force Research Laboratory/Air Vehicles Directorate).

\section{References}

\footnotetext{
${ }^{1}$ Bolender, M. A. and Doman, D. B., "Nonlinear Longitudinal Dynamical Model of an Air-Breathing Hypersonic Vehicle," Journal of Spacecraft and Rockets, Vol. 44, No. 2, March-April 2007, pp. 374-387.

${ }^{2}$ Chavez, F. R. and Schmidt, D. K., "Analytical Aeropropulsive/Aeroelastic Hypersonic-Vehicle Model with Dynamic Analysis," Journal of Guidance, Control, and Dynamics, Vol. 17, No. 6, March-April 2007, pp. 1308-1319.

${ }^{3}$ Anderson, Jr., J. D., Hypersonic and High-Temperature Gas Dynamics, McGraw-Hill, 1989. 1994.

${ }^{4}$ Bertin, J. J., Hypersonic Aerothermodynamics, American Institute of Aeronautics and Astronautics, Reston, Virgina,

${ }^{5}$ Minkowycz, W. J. and Sparrow, E. M., editors, Advances in Numerical Heat Transfer, Vol. II, Taylor \& Francis, New York, 2000.

${ }^{6}$ Guyan, R. J., "Reduction of Stiffness and Mass Matrices," AIAA Journal, Vol. 3, No. 2, 1965, pp. 380.

${ }^{7}$ Craig, Jr., R. R. and Bampton, M. C. C., "Coupling of Substructures for Dynamic Analysis," AIAA Journal, Vol. 6, No. 7, July 1968, pp. 1313-1319.

${ }^{8}$ Botto, D., Gola, S., Troncarelli, M. M., and Pasquero, G., "Component Modes Synthesis Applied to a Thermal Transient Analysis of a Turbine Disc," Worldwide Aerospace Conference and Technology Showcase, Toulouse, 2002.

${ }^{9}$ Botto, D., Zucca, S., Gola, M. M., and Salvano, S., "A Method for on Line Temperature Calculation of Aircraft Engine Turbine Discs," ASME Turbo Expo, Amsterdam, 2002.

${ }^{10}$ Botto, D., Zucca, S., and Gola, M. M., "A Methodology for On-Line Calculation of Temperature and Thermal Stress Under Non-Linear Boundary Conditions," Int. J. Press. Vess. Pip., Vol. 80, No. 1, 2003, pp. 21-29.

${ }^{11}$ Botto, D., Zucca, S., and Gola, M. M., "Reduced-Order Models for the Calculation of Thermal Transients of Heat Conduction/Convection FE Models," Journal of Thermal Stresses, Vol. 30, No. 8, August 2007, pp. 819-839.

${ }^{12}$ Petit, D., Hachette, R., and Veyret, D., "A Modal Identification Method to Reduce a High-Order Model: Application to Heat Conduction Modelling," International Journal of Modelling \& Simulation, Vol. 17, No. 3, 1997, pp. 242-250.

${ }^{13}$ Videcoq, E. and Petit, D., "Model Reduction for the Resolution of Multidimensional Inverse Heat Conduction Problems," Int. J. Heat Mass Transfer, Vol. 44, No. 10, 2001, pp. 1899-1911.

${ }^{14}$ Girault, M. and Petit, D., "Identification methods in nonlinear heat conduction. Part I: model reduction," Int. Journal of Heat Mass Transfer, Vol. 48, No. 1, 2005, pp. 105-118.

${ }^{15}$ Girault, M. and Petit, D., "Identification methods in nonlinear heat conduction. Part II: Inverse Problem Using a Reduced Model," Int. Journal of Heat Mass Transfer, Vol. 48, No. 1, 2005, pp. 119-133.

${ }^{16}$ Shore, C. P., "Reduction Method for Thermal Analysis of Complex Aerospace Structure," Technical Paper NASA-TP2373, Langley Research Center, Jan. 1985.

${ }^{17}$ Balakrishnan, N. V., Nonlinear Transient Thermal Analysis by the Force-Derivative Method, Ph.D. thesis, Old Dominion University, Norfolk, Virginia, 1995.

${ }^{18}$ Camarda, C. J. and Haftka, R. T., "Development of Higher-Order Modal Methods for Transient Thermal and Structural Analysis," Tech. Rep. NASA TM-101548, NASA Langley Research Center, February 1989.

${ }^{19}$ Camarda, C. J., "Development of Advanced Modal Methods for Calculating Transient Thermal and Structural Response," Tech. Rep. NASA TM-104102, NASA Langley Research Center, December 1991.

${ }^{20}$ Coutinho, A. L. G. A., Landau, L., Wrobel, L. C., and Ebecken, N. F. F., "Modal Solution of Transient Heat Conduction Utilizing Lanczos Algorithm," International Journal for Numerical Methods in Engineering, Vol. 28, No. 1, 1989, pp. 13-25.

${ }^{21}$ Cardona, A. and Idelsohn, S., "Solution of Non-Linear Thermal Transient Problems by a Reduction Method," International Journal for Numerical Methods in Engineering, Vol. 23, No. 6, 1986, pp. 1023-1042.

${ }^{22}$ Nour-Omid, B., "Lanczos Method for Heat Conduction Analysis," International Journal for Numerical Methods in Engineering, Vol. 24, No. 1, 1987, pp. 251-262.

${ }^{23}$ Camarda, C. J., Haftka, R. T., and Riley, M. F., "An Evaluation of Higher-Order Modal Methods for Calculating Transient Structural Response," Computers 83 Structures, Vol. 27, No. 1, 1987, pp. 89-101.

${ }^{24}$ Holmes, P., Lumley, J., and Berkooz, G., Turbulence, Coherent Structures, Dynamical Systems and Symmetry, Cambridge University Press, 1996.

${ }^{25}$ Liang, Y. C., Lee, H. P., Lim, S. P., Lin, W. Z., Lee, K. H., and Wu, C. G., "Proper Orthogonal Decomposition and its Applications-Part I: Theory," Journal of Sound and Vibration, Vol. 252, No. 3, 2002, pp. 527-544.

${ }^{26}$ Lumley, J. L., "The Structure of Inhomogeneous Turbulent Flows," Atmospheric Turbulence and Radio Wave Propagation, edited by A. Yaglon and V. Tatarsky, Nauka, Moscow, 1967, pp. 166-178.
} 
${ }^{27}$ Krysl, P., Lall, S., and Marsden, J. E., "Dimensional Model Reduction in Non-Linear Finite Element Dynamics of Solids and Structures," International Journal for Numerical Methods in Engineering, Vol. 51, 2001, pp. 479-504.

${ }^{28}$ Placzek, A., Tran, D., and Ohayon, R., "Hybrid proper orthogonal decomposition formulation for linear structural dynamics," Journal of Sound and Vibration, Vol. 318, No. 4-5, December 2008, pp. 943-964.

${ }^{29}$ Hall, K. C., Thomas, J. P., and Dowell, E. H., "Proper Orthogonal Decomposition Technique for Transonic Unsteady Aerodynamic Flows," AIAA Journal, Vol. 38, No. 10, October 2000, pp. 1853-1862.

${ }^{30}$ Atwell, J. A. and King, B. B., "Proper Orthogonal Decomposition for Reduced Basis Feedback Controllers for Parabolic Equations," Mathematical and Computer Modelling, Vol. 33, 2001, pp. 1-19.

${ }^{31}$ Bialecki, R. A., Kassab, A. J., and Fic, A., "Proper Orthogonal Decomposition and Modal Analysis for Acceleration of Transient FEM Thermal Analysis," International Journal for Numerical Methods in Engineering, Vol. 62, No. 6, 14 Feb. 2005, pp. $774-797$.

${ }^{32}$ Bialecki, R. A., Kassab, A. J., and Fic, A., "Reduction of the Dimensionality of Transient FEM Solutions Using Proper Orthogonal Decomposition," Proceedings of the 36th AIAA Thermophysics Conference, Orlando, Florida, AIAA 2003-4207.

${ }^{33}$ Fic, A., Bialecki, R. A., and Kassab, A. J., "Solving Transient Nonlinear Heat Conduction Problems by Proper Orthogonal Decomposition and the Finite-Element Method," Numerical Heat Transfer, Part B: Fundamentals, Vol. 48, No. 2, Aug. 2005, pp. 103-124.

${ }^{34}$ Önder Efe, M. and Özbay, H., "Proper Orthogonal Decomposition for Reduced Order Modeling: 2D Heat Flow," Proceedings of the 2003 IEEE Conference on Control Applications, Istanbul, Turkey, 2003.

${ }^{35}$ Park, H. M., Chung, O. Y., and Lee, J. H., "On the solution of inverse heat transfer problem using the Karhunen-Loève Galerkin Method," Int. J. Heat Mass Transfer, Vol. 42, No. 1, 1999, pp. 127-142.

${ }^{36}$ Park, H. M. and Jung, W. S., "The Karhunen-Loève Galerkin Method for the Inverse Natural Convection Problems," Int. J. Heat Mass Transfer, Vol. 44, No. 1, 2001, pp. 155-167.

${ }^{37}$ Ostrowski, Z., Bialecki, R. A., and Kassab, A. J., "Estimation of Constant Thermal Conductivity by Use of Proper Orthogonal Decomposition," Computational Mechanics, Vol. 37, No. 1, Dec. 2005, pp. 52-59.

${ }^{38}$ Falkiewicz, N. J. and Cesnik, C. E. S., "A Reduced-Order Modeling Framework for Integrated Thermo-Elastic Analysis of Hypersonic Vehicles," Proceedings of the 50th AIAA/ASME/ASCE/AHS/ASC Structures, Structural Dynamics, and Materials Conference, Palm Springs, California, AIAA 2009-2308.

${ }^{39}$ Sirovich, L., "Turbulence and Dynamics of Coherent Structures, Part I: Coherent Structures," Quarterly of Applied Mathematics, Vol. XLV, 1987, pp. 561-571.

${ }^{40}$ Gunzburger, M. and Peterson, J., "Reduced-Order Modeling of Complex Systems with Multiple System Parameters," Large-Scale Scientific Computing, 5th International Conference, Sozopol, Bulgaria, edited by I. Lirkov, S. Margenov, and J. Wasniewski, Vol. LNCS 3743, Springer Verlag, 2006, pp. 15-27.

${ }^{41}$ Trefethen, L. N. and Bau, III, D., Numerical Linear Algebra, Society for Industrial and Applied Mathematics, 1997.

${ }^{42}$ Heath, M. T., Scientific Computing : An Introductory Survey, McGraw-Hill, 2nd ed., 2002.

${ }^{43}$ Chatterjee, A., "An introduction to the proper orthogonal decomposition," Current Science, Vol. 78, No. 7, April 2000, pp. 808-817.

${ }^{44}$ Huebner, K., Dewhirst, D., Smith, D., and Byrom, T., The Finite Element Method for Engineers, John Wiley \& Sons, Inc., 4th ed., 2001.

${ }^{45}$ Wood, W. L. and Lewis, R. W., "A Comparison of Time Marching Schemes for the Transient Heat Conduction Equation," Int. J. Numer. Methods Eng., Vol. 9, No. 3, 1975, pp. 679-689.

${ }^{46}$ Hirsch, C., Numerical Computation of Internal \& External Flows, Butterworth-Heinemann, 2nd ed., 2007.

${ }^{47}$ Hughes, T. J. R., "Unconditionally Stable Algorithms for Nonlinear Heat Conduction," Computer Methods in Applied Mechanics and Engineering, Vol. 10, 1977, pp. 135-139.

${ }^{48}$ Oppenheimer, M. W. and Doman, D. B., "A Hypersonic Vehicle Model Developed with Piston Theory," Proceedings of the 2006 AIAA Atmospheric Flight Mechanics Conference, Keystone, Colorado, AIAA 2006-6637.

${ }^{49}$ Pendleton, E., Moster, G., and Farmer, M., "Transonic Aeroelastic Models of Hypersonic Highly Swept Lifting Surfaces: Design, Analyses, and Test," Proceedings of the 35th AIAA/ASME/ASCE/AHS/ASC Structures, Structural Dynamics, and Materials Conference, Hilton Head, South Carolina, AIAA 1994-1489-574.

${ }^{50}$ Pendleton, E., Moster, G., and Keller, D., "Transonic Aeroelastic Models of Highly Swept Hypersonic Lifting Surfaces," Journal of Aircraft, Vol. 32, No. 6, Nov. - Dec. 1995, pp. 1169-1176.

${ }^{51}$ Spain, C. V., Zeiler, T. A., Bullok, E. P., and Hodge, J. S., "A Flutter Investigation of All-Moveable NASP-Like Wings at Hypersonic Speeds," Proceedings of the 34th AIAA/ASME/ASCE/AHS/ASC Structures, Structural Dynamics, and Materials Conference, La Jolla, California, AIAA-93-1315.

${ }^{52} \mathrm{McNamara}$, J. J., Aeroelastic and Aerothermoelastic Behavior of Two and Three Dimensional Lifting Surfaces in Hypersonic Flow, Ph.D. thesis, University of Michigan, Ann Arbor, Michigan, 2005.

${ }^{53}$ Shih, P., Prunty, J., and Mueller, R., "Thermostructural Concepts for Hypervelocity Vehicles," Journal of Aircraft, Vol. 28, No. 5, May 1991, pp. 337-345.

${ }^{54}$ Myers, D., Martin, C., and Blosser, M., "Parametric Weight Comparison of Advanced Metallic, Ceramic Tile, and Ceramic Blanket Thermal Protection Systems," Tech. Rep. NASA TM-210289, Langley Research Center, June 2000.

${ }^{55}$ Orton, G. and Scuderi, L., "A Hypersonic Cruiser Concept for the 21st Century," Proceedings of the 1998 World Aviation Conference, Anaheim, California, AIAA 1998-5525.

${ }^{56}$ Wieting, A., Dechaumphai, P., Bey, K., Thornton, E., and Morgan, K., "Application of Integrated Fluid-ThermalStructural Analysis Methods," Thin-Walled Structures, Vol. 11, No. 1-2, 1991, pp. 1-23.

${ }^{57}$ Leatherwood, J., Clevenson, S., Powell, A., and Daniels, E., "Acoustic Testing of High-Temperature Panels," Journal of Aircraft, Vol. 29, No. 6, Nov. - Dec. 1992, pp. 1130-1136. 
${ }^{58}$ Blevins, R., Bofilios, D., Holehouse, I., Hwa, V., Tratt, M., Laganelli, A., Pozefsky, P., and Pierucii, M., "Thermo-VibroAcoustic Loads and Fatigue of Hypersonic Flight Vehicle Structure," Tech. Rep. AFRL-RB-WP-TR-2009-3139, Air Force Research Laboratory, June 2009.

${ }^{59}$ Ellis, D., Pagel, L., and Schaeffer, D., "Design and Fabrication of a Radiative Actively Cooled Honeycomb Sandwich Structural Panel for a Hypersonic Aircraft," Tech. Rep. NASA-CR-2957, NASA, March 1978.

${ }^{60}$ Ko, W. and Fields, R., "Thermal Stress Analysis of Space Shuttle Orbiter Subjected to Reentry Aerodynamic Heating," Tech. Rep. NASA-TM-88286, NASA, Oct. 1987.

${ }^{61}$ Leyens, C. and Peters, M., Titanium and Titanium Alloys, Wiley-VHC, 2003.

${ }^{62}$ Noor, A. K., editor, Structures Technology: Historical Perspective and Evolution, American Institute of Aeronautics and Astronautics, 1998.

${ }^{63}$ Department of Defense, Military Handbook - MIL-HDBK-5H: Metallic Materials and Elements for Aerospace Vehicle Structures, December 1998.

${ }^{64}$ Welsch, G., Boyer, R., and Collings, E. W., Materials Properties Handbook: Titanium Alloys, ASM International, 1994.

65 "Titanium IMI 834," http://http://www.matweb.com/search/DataSheet.aspx?MatGUID= a74096c99aa6486382a9c9e1be0883c4\&ckck=1, Last Accessed 9/3/2009.

${ }^{66}$ Craig, R. R. and Kurdila, A. J., Fundamentals of Structural Dynamics, John Wiley \& Sons, Inc., 2 nd ed., 2006.

${ }^{67}$ Falkiewicz, N. J., Cesnik, C. E. S., Bolender, M. A., and Doman, D. B., "Thermoelastic Formulation of a Hypersonic Vehicle Control Surface for Control-Oriented Simulation," Proceedings of the 2009 AIAA Guidance, Navigation, and Control Conference, Chicago, Illinois, AIAA 2009-6284.

${ }^{68}$ Holman, J. P., Heat Transfer, McGraw-Hill, Inc., 8th ed., 1997.

${ }^{69}$ Eckert, E. R. G., "Survey on Heat Transfer at High Speeds," Technical Report ARL 189, Aeronautical Research Laboratory, University of Minnesota, Minneapolis, MN, December 1961.

${ }^{70}$ Lighthill, M., "Oscillating Airfoils at High Mach Number," Journal of the Aeronautical Sciences, Vol. 20, No. 6, June 1953, pp. 402-406.

${ }^{71}$ Ashley, H. and Zartarian, G., "Piston Theory - A New Aerodynamic Tool for the Aeroelastician," Journal of the Aeronautical Sciences, Vol. 23, No. 12, 1956, pp. 1109-1118.

${ }^{72}$ Oppenheimer, M. W., Skujins, T., Bolender, M. A., and Doman, D. B., "A Flexible Hypersonic Vehicle Model Developed with Piston Theory," Proceedings of the 2007 AIAA Atmospheric Flight Mechanics Conference, Hilton Head, South Carolina, AIAA 2007-6396.

${ }^{73}$ Mei, C., Motagaly, K. A., and Chen, R., "Review of Nonlinear Panel Flutter at Supersonic and Hypersonic Speeds," Applied Mechanics Review, Vol. 52, No. 10, October 1999, pp. 321-332.

${ }^{74}$ McNamara, J. J. and Friedmann, P. P., "Aeroelastic and Aerothermoelastic Analysis of Hypersonic Vehicles: Current STatus and Future Trends," Proceedings of the 48th AIAA/ASME/ASCE/AHS/ASC Structures, Structural Dynamics, and Materials Conference, Honolulu, Hawaii, AIAA 2007-2013.

${ }^{75}$ Anderson, J. D., Modern Compressible Flow: With Historical Perspective, McGraw-Hill, Boston, 3rd ed., 2003.

${ }^{76}$ Thuruthimattam, B. J., Friedmann, P. P., McNamara, J. J., and Powell, K. G., "Modeling Approaches to Hypersonic Aeroelasticity," 2002 ASME International Mechanical Engineering Congress and Exposition, New Orleans, Louisiana, November IMECE/2002-32943. 